\title{
Comparison between ray-tracing simulations and bi- directional transmission measurements on prismatic glazing
}

\author{
Marilyne Andersen*,†, Michael Rubin**, Jean-Louis Scartezzini* \\ *Solar Energy and Building Physics Laboratory LESO-PB, Swiss Federal Institute of Technology EPFL, 1015 \\ Lausanne, Switzerland \\ **Lawrence Berkeley National Laboratory, University of California, 1 Cyclotron Road, MS 2-300, Berkeley CA \\ 94720-8134, USA
}

\begin{abstract}
Evaluation of solar heat gain and daylight distribution through complex window and shading systems requires the determination of the Bi-directional Transmission Distribution Function (BTDF). Measurement of BTDF can be time-consuming, and inaccuracies are likely because of physical constraints and experimental adjustments. A general calculation methodology, based on more easily measurable component properties, would be preferable and would allow much more flexibility. In this paper, measurements and calculations are compared for the specific case of prismatic daylight-redirecting panels. Measurements were performed in a photogoniometer equipped with a digital-imaging detection system. A virtual copy of the photogoniometer was then constructed with commercial ray-tracing software. For the first time, an attempt is made to validate detailed bi-directional properties for a complex system by comparing an extensive set of experimental BTDF data with ray-tracing calculations. The results generally agree under a range of input and output angles to a degree adequate for evaluation of glazing systems. An analysis is presented to show that the simultaneously measured diffuse and direct components of light transmitted by the panel are properly represented. Calculations were also performed using a more realistic model of the source and an ideal model of the detector. Deviations from the photogoniometer model were small and the results were similar in form. Despite the lack of an absolute measurement standard the good agreement in results promotes confidence in both the photogoniometer and in the calculation method.
\end{abstract}

Keywords: ray-tracing calculations, computer simulation, daylighting, photometry, Bi-directional Transmission Distribution Function (BTDF), empirical validation, advanced fenestration systems.

\section{Introduction}

Energy savings objectives and improvement of visual comfort in buildings has lead to a growing need for accurate bi-directional transmission data for advanced windows, shading systems and daylight redirecting devices. Specialized experimental systems have been developed to measure the Bi-directional (light) Distribution Function. This BTDF function is defined as "the quotient of the luminance of a surface element in a given direction, by the illuminance incident on the sample" (CIE, 1977), and is expressed in $\left[\mathrm{cd} \mathrm{m}^{-2} \mathrm{~lx}^{-1}\right]$ or $\left[\mathrm{sr}^{-1}\right]$.

\footnotetext{
${ }^{\dagger}$ Author to whom correspondence should be addressed. Tel.: +41-21-693-45-51; fax: +41-21-693-27-22; e-mail: marilyne.andersen@epfl.ch
} 
The usual way to measure bi-directional transmission functions is based on a point-per-point mapping of the emerging hemisphere with a device-specific detector (Papamichael, 1988; Murray-Coleman and Smith, 1990; Apian-Bennewitz, 1994; Bakker and van Dijk, 1995; Breitenbach and Rosenfeld, 1998; Aydinli, 1999). The operating principle of the photogoniometer developed at the LESO-PB/EPFL is different: it is based on the observation of a mobile projection screen, from which the transmitted light is reflected into a calibrated CCD camera (Andersen et al., 2001). This technique allows a considerable reduction of the time needed to perform BTDF measurements. The entire light distribution is characterized within 6 screen rotations instead of hundreds or more likely thousands of detector movements through a comparable number of positions. Also, this approach gives a continuous knowledge of the whole transmission space, averaged into finite zones, instead of discrete transmission assessments that need to be interpolated.

There is however a lack of standards for absolute determination of the optical properties of complex glazing systems. Consequently, validation of BTDF data obtained with photogoniometric measurements has so far been restricted to two limited possibilities: (a) Perform BTDF measurements on simple glazing or systems, which can be assessed in a standardized spectrometer or analytically calculated (Murray-Coleman and Smith, 1990; Andersen et al., 2000); (b) Calculate the global transmittance values from an integration of BTDFs over the emerging hemisphere and compare them to Ulbricht sphere measurements (Murray-Coleman and Smith, 1990; Apian-Bennewitz, 1994; Breitenbach and Rosenfeld, 1998; Andersen et al., 2000; van Dijk, 2001). These methods are reliable, and obtaining good results in such comparisons is promising for the BTDF assessment accuracy. However, they cannot be considered as sufficient to prove that individual BTDF values are accurate enough for fenestration systems of arbitrary complexity. Likewise, a BTDF comparison from one facility to another cannot provide definitive conclusions yet, as neither of the two datasets could be considered as better than the other.

The use of ray-tracing techniques can provide a general method for evaluating complex systems in full detail and add a point of comparison to bi-directional measurements. The combination of experimental and computational methods will increase flexibility and efficiency by restricting the experimental part to the essential measurements only, i.e. the transmission and reflection properties of unknown component coatings or materials. Computational methods have already been used or developed for the assessment of complex glazing (see e.g. Compagnon, 1994; Mitanchey et al., 1995; Molina et al., 1995; Campbell, 1998; Kuhn et al., 2000), and have proven their usefulness and potentialities. However, they have so far never served extensively as a basis for BTDF measurement comparisons, as presented here.

In this paper, experimental conditions for BTDF characterization with the digital imaging-based photogoniometer developed at the Swiss Federal Institute of Technology (EPFL) are reproduced virtually with the commercial forward ray-tracer TRACEPRO ${ }^{\circledR}$ for two acrylic prismatic panels. The latter have been chosen as a validation example because they consist of a material of wellknown refraction coefficients, thus easily handled by the software applying Snell-Descartes' law, whereas at the same time they present complex transmission features because of the multiple inside reflections and interactions between the gratings.

\footnotetext{
* TracePro®, v. 2.3 \& 2.4, Lambda Research Corporation.
} 


\section{Description of BTDF experimental assessment method}

The principle of the bi-directional photogoniometer constructed at the Solar Energy and Building Physics Laboratory (LESO-PB / EPFL) is based on digital imaging techniques. The light transmitted from the sample is reflected by a diffusing triangular panel towards a charge-coupled device (CCD) camera, which provides a picture of the whole screen, as illustrated by Fig. 1. The incident direction $\left(\theta_{1}, \phi_{1}\right)$ is determined by inclining the device (and hence the sample plane) around a horizontal axis at altitude $\theta_{1}$ and by rotating the sample around its normal to reach azimuth $\phi_{1}$, the light source remaining fixed.

The CCD camera is used as a multiple-points luminance-meter, and has been calibrated accordingly (Andersen et al., 2001); a relation between the pixel's coordinates on the image and the angular direction $\left(\theta_{2}, \phi_{2}\right)$ they correspond to has been established as a function of the sample thickness and the incident azimuth value $\phi_{1}$ (as the referential rotates with the sample for nonzero azimuths $\phi_{1}$ ), thanks to the use of matrix calculations (Andersen, 2001). After six $60^{\circ}$ rotations of the screen-camera system (with image capture, calibration and processing at each position), the transmitted light distribution is fully known. "Screen" luminance values can then be converted into BTDF data according to equation (1), where distance and light tilting effects are compensated. Details can be found in Andersen et al. (2001) and Andersen (2002).

$\operatorname{BTDF}\left(\theta_{1}, \phi_{1}, \theta_{2}, \phi_{2}\right)\left[\frac{c d}{m^{2} l x}\right]=\frac{\pi}{\rho} \cdot \frac{d^{2}\left(\theta_{2}, \phi_{2}\right)}{A \cdot \cos \theta_{2} \cdot \cos \alpha} \cdot \frac{L_{\text {screen }}\left(\theta_{1}, \phi_{1}, \theta_{2}, \phi_{2}\right)}{E_{1}\left(\theta_{1}\right)}$

where

$\left(\theta_{1}, \phi_{1}\right)$ : Polar co-ordinates of incoming light flux $\left[{ }^{\circ}\right]$

$\left(\theta_{2}, \phi_{2}\right)$ : Polar co-ordinates of emerging (transmitted) light flux $\left[^{\circ}\right]$

$\rho$ : Reflection factor of projection screen [-]

$\mathrm{d}$ : Distance from sample center to screen along direction $\left(\theta_{2}, \phi_{2}\right)[\mathrm{m}]$

A: Illuminated area of sample $\left[\mathrm{m}^{2}\right]$

$\alpha$ : Angle between the normal to the screen and the direction $\left(\theta_{2}, \phi_{2}\right)\left[^{\circ}\right]$

$\mathrm{L}_{\text {screen }}\left(\theta_{1}, \phi_{1}, \theta_{2}, \phi_{2}\right)$ : Luminance of the projection screen area associated to the direction $\left(\theta_{2}, \phi_{2}\right)$ $\left[\mathrm{cd} \mathrm{m}^{-2}\right]$

$\mathrm{E}_{1}\left(\theta_{1}\right)$ : Illuminance of the fenestration material due to the incoming light flux [lx].

Fig. 1. Diagram of the LESO-PB bi-directional photogoniometer showing the use of a CCD camera as a multiple-points luminance-meter.

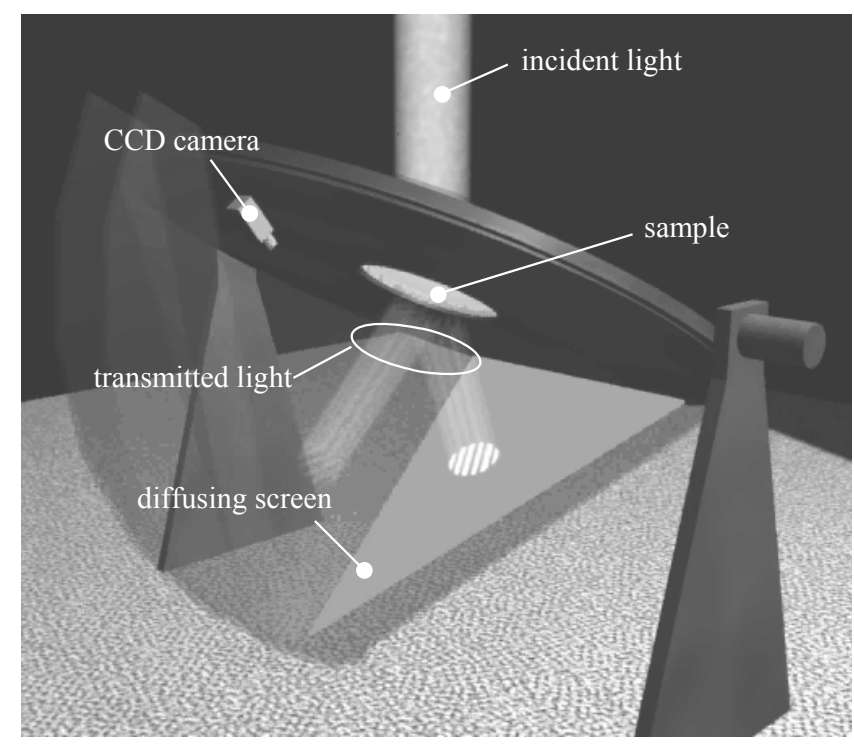


In order to determine BTDF values according to a regular output resolution $\left(\Delta \theta_{2}, \Delta \phi_{2}\right)$, outgoing zones have to be defined around the considered directions $\left(\theta_{2}, \phi_{2}\right)$. The luminances due to the transmitted light flux being measured on a projection screen, the latter must be divided into a grid of zones depending on the desired output resolution. The size of the zones (i.e. the number of comprised pixels) are hence inversely proportional to the number of analysed directions, which are bound to the output resolution.

This approach allows the investigation of the whole transmission hemisphere without any unexplored area. Resolution-dependent BTDF values result from an average over a certain outgoing zone, limited by $\left(\phi_{2}-1 / 2 \Delta \phi_{2} ; \phi_{2}+1 / 2 \Delta \phi_{2}\right)$ in azimuth and by $\left(\theta_{2}-1 / 2 \Delta \theta_{2} ; \theta_{2}+1 / 2 \Delta \theta_{2}\right)$ in altitude for each outgoing direction. For non-Lambertian materials, such BTDF data will therefore present differences with point-per-point photogoniometric measurements, where a new output resolution only affects the shift between two measurement positions, and not the BTDF value itself obtained for a given direction $\left(\theta_{2}, \phi_{2}\right)$. On the other hand, the conventional method cannot avoid a loss of information for the in-between regions. The discretization of the output hemisphere into zones representing average light emergence around particular directions $\left(\theta_{2}, \phi_{2}\right)$ has thus the important advantage of providing a continuous characterization of the transmitted light distribution. This is particularly critical when the latter presents narrow luminance peaks as in the case of prismatic panels.

Other major advantages of digital-imaging techniques are the great reduction in time to complete the full set of measurements, and accuracy holding even for high luminance dynamics.

It must be noted that this assessment method leads to BTDF average values not only related to the direction of the emerging rays, but also to the angular areas where these rays are detected, whose location is determined by the considered direction and its size by the output resolution. This difference has however a negligible impact on the monitored data as long as the distance from the sample to the detector (screen) is large compared to the sample size, a factor of 10 being accepted as reasonable. Besides, the angular resolution is always chosen according to the sample size, in order to remain consistent with the possible divergence of rays reaching a given point.

Experimental assessment of BTDFs and corresponding ray-tracing calculations were compared for prismatic panels, revealing a specular transmission with directional changes due to refraction. Formally, a BTDF is only defined for diffuse transmission (CIE, 1977); however, as pointed out by Murray-Coleman and Smith (1990), BTDFs are capable of describing specular as well as diffuse transmission. In the specular case, BTDFs present a finite value determined by the incident angle, the transmittance, and the source solid angle, except in the limit of a vanishingly small source solid angle, where a specular BTDF will approach infinity. Although the analytical expressions of BTDFs differ whether they are related to specular or diffuse light, their common assessment can be accepted under certain conditions, presented in appendix A. These conditions can be considered as reasonably approached for the particular data acquisition methodology developed for the formerly described digital imaging-based photogoniometer, as detailed in the appendix and shown by the ideal situation comparison exposed in $\S 6$. 


\section{Characterization parameters and sample description}

Two acrylic prismatic panels, manufactured by Siemens AG, have been selected for this study among the samples characterized with the bi-directional photogoniometer: one presents symmetric gratings of slope $45^{\circ}$ (see Fig. 2A) and the other asymmetric gratings of slope $42^{\circ}$ and $5^{\circ}$ (Fig. 2B). These particular complex glazing materials were chosen because of their combination of simplicity in virtual representation and complexity in light transmission. Their geometric characteristics are well defined and can be determined at a macroscopic level. In addition, their physical properties can be easily described in a simulation program, acrylic being a common material with well-known wavelength-dependent indices of refraction. At the same time, they present complex transmission features because of the multiple internal reflections and interactions between adjacent gratings.

The dimensions of the two panels are respectively: height (vertical dimension) $90 \mathrm{~mm}$, length (horizontal dimension) $200 \mathrm{~mm}$ and thickness $12 \mathrm{~mm}$ for the symmetric panel (height of individual grating $=15 \mathrm{~mm}$ ); height $195 \mathrm{~mm}$, length $200 \mathrm{~mm}$ and thickness $12 \mathrm{~mm}$ for the asymmetric panel (height of individual grating $=7 \mathrm{~mm}$, last grating incomplete).

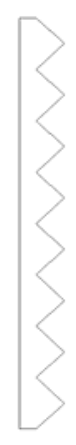

A

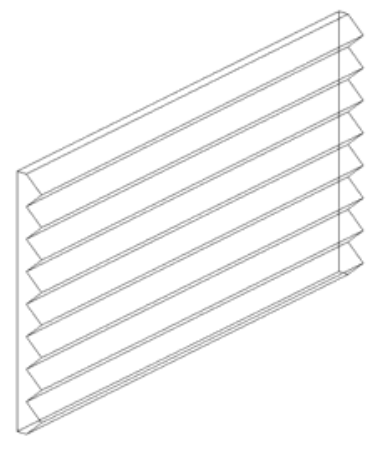

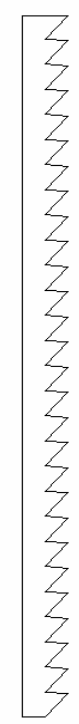

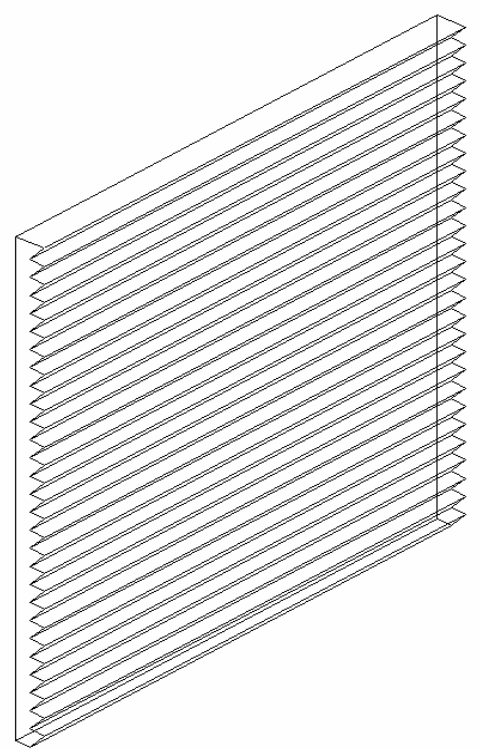

B

Fig. 2. Section view and parallel perspective of the prismatic panels used for BTDF assessment validation. (A) Symmetric panel, gratings $45^{\circ}$. (B) Asymmetric panel, gratings $42^{\circ}$ and $5^{\circ}$.

The origin of the co-ordinate system is placed on the panel (see Fig. 3). Directions are defined by spherical co-ordinates: altitude angle $\theta_{\mathrm{i}}$ comprised between $0^{\circ}$ and $90^{\circ}$, and azimuth angle $\phi_{\mathrm{i}}$ comprised between $0^{\circ}$ and $360^{\circ}$, where index i indicates whether it is related to the incident $(\mathrm{i}=$ $1)$ or transmitted $(i=2)$ direction. The respective base planes are the external (on incidence side) and internal (on emerging side) sample interfaces. 
A full set of BTDF data was generated experimentally for the symmetric panel (with flat face on incident side), according to the 145 incident directions of the sky luminance mapping proposed by Tregenza (1987) within the IDMP international programme; this set is considered as a standard for photogoniometric data at the international level (Aydinli, 1999). The maximum set of incident directions has been reduced because of the sample symmetries, leading to 42 relevant incident directions for the $45^{\circ}$ gratings panel. This sample was characterized for six additional incident directions, defined by: $\theta_{1}=20^{\circ}$ and $40^{\circ}, \phi_{1}=0^{\circ}, 45^{\circ}$ and $90^{\circ}$.

The asymmetric panel was analysed for gratings on both incident and emerging sides (the $5^{\circ}$ slope upwards, as in Fig. 2B), and along azimuthal planes $\phi_{1}=0^{\circ}, 90^{\circ}$ and $270^{\circ}$ with a regular altitude increment of $10^{\circ}$.

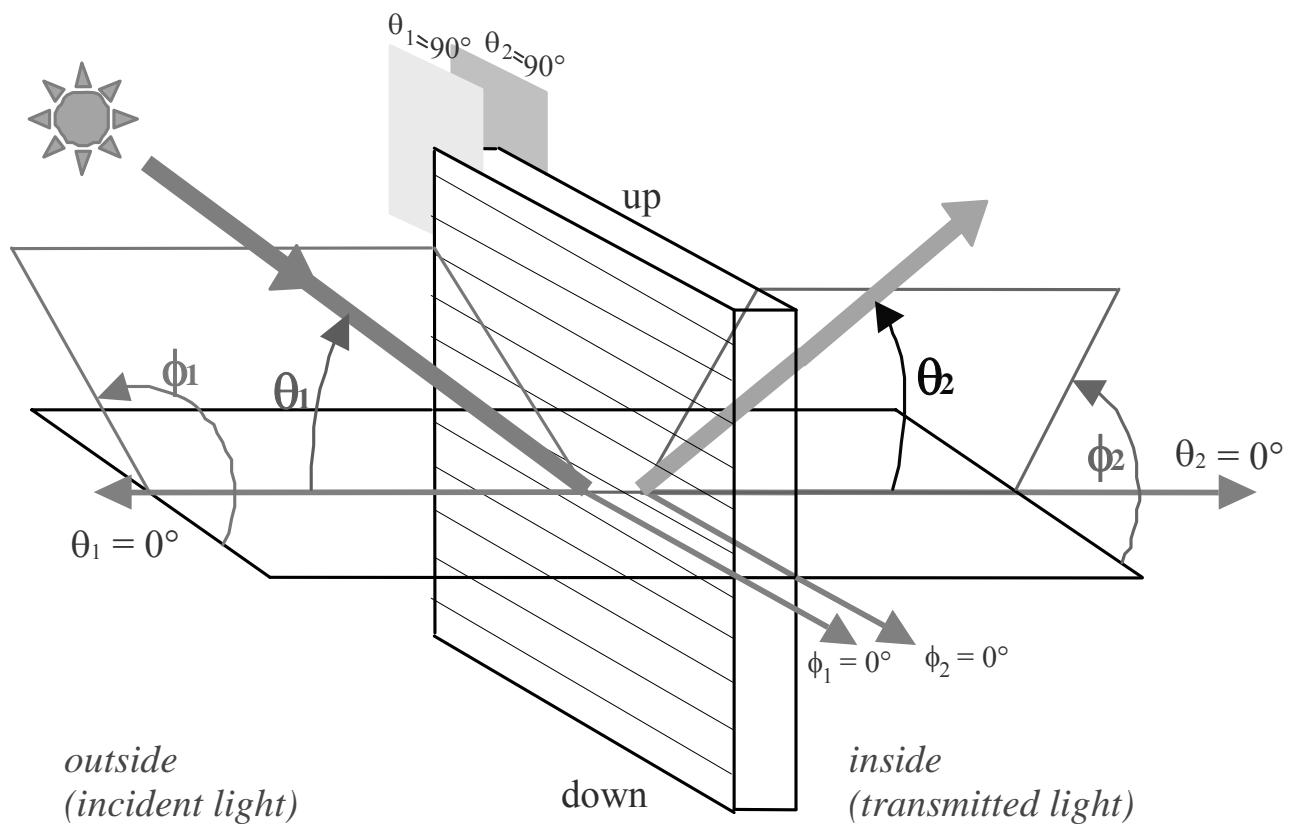

Fig. 3. Spatial referential with regard to the sample orientation for incident (index 1) and transmitted (index 2) directions.

The light source consists of a HMI $2.5 \mathrm{~kW}$ discharge lamp with a Fresnel lens. Its spectrum is given in Fig. 4; as detailed in Andersen et al. (2000), the uniformity of the incident radiation has been checked to present a relative mean deviation lower than $1.8 \%$ over the sample area, and the analysis of the collimation of the beam reaching the latter has lead to a half angle of $0.4^{\circ}$.

The detection screen is a triangle of base $115 \mathrm{~cm}$ and height $152.1 \mathrm{~cm}$, fixed on a rotating ring with an angle of $49.1^{\circ}(=$ atan $2 / \sqrt{ } 3)$ in order that its projection on the sample plane represents an equilateral triangle. Its base plane is slightly shifted out from the incident base plane $(7.5 \mathrm{~cm}$ between the two).

The output resolution is equal for both samples $\left(\Delta \theta_{2}=5^{\circ}, \Delta \phi_{2}=5^{\circ}\right)$. Because of the samples physical dimension, the illuminated area for the symmetric panel was restricted to a disk of diameter $6 \mathrm{~cm}$ with an opaque diaphragm, whereas the asymmetric panel was measured with a $10 \mathrm{~cm}$ diaphragm. 


\section{Virtual reproduction of BTDF measurements with ray-tracing calculations}

In order to validate the measured BTDF values, the commercial ray-tracing simulation software TRACEPRo ${ }^{\circledR}$, based on Monte Carlo calculations, was used to replicate the experimental conditions. All the optical components (light source, spectrum, sample, detection system) were therefore simulated with geometric and material characteristics as close as possible to the reality. Of course, the virtual BTDF values are ideal in regards to what the experimental set-up can generate: in addition to the inevitable uncertainties due to the components physical nature, the model was built according to the simplification hypotheses formulated by Compagnon (1994), neglecting light dispersion and absorption inside the prismatic material, edge effects or dust, and shape imperfections (like rounded grating edges appearing for any manufactured element). These simplifications were nevertheless taken into account within the error calculation procedure (see $\S$ $5)$.

The simulation model had to follow important constraints, such as:

- The incident source must be of same angular spread as the real one; a set of wavelengths representative of its spectrum has to be determined; the source has to be positioned in order to reproduce the same incident directions as the ones analysed.

- A sample of same geometric and physical (acrylic) properties as the one measured has to be modelled; the sample area exposed to light has to fit the illuminated surface during experimental characterization.

- A detection screen of same geometry as the one used physically for the measurement facility and separated into the same pattern of zones (solid angles around outgoing directions, see $\S 2$ ) has to be modelled.

For each prismatic panel, five representative incidences were selected amongst the full set of 98 incident directions that were characterized experimentally. The corresponding angular couples $\left(\theta_{1}, \phi_{1}\right)$ are: for the symmetric panel (flat face on incident side), $\left(40^{\circ}, 90^{\circ}\right),\left(60^{\circ}, 90^{\circ}\right),\left(24^{\circ}, 30^{\circ}\right)$, $\left(40^{\circ}, 45^{\circ}\right)$ and $\left(60^{\circ}, 75^{\circ}\right)$; for the asymmetric panel, $\left(20^{\circ}, 0^{\circ}\right)$ and $\left(40^{\circ}, 90^{\circ}\right)$ for the flat face on incident side, $\left(0^{\circ}, 0^{\circ}\right),\left(10^{\circ}, 90^{\circ}\right)$ and $\left(20^{\circ}, 270^{\circ}\right)$ for the gratings on the incident side.

\subsection{Virtual components}

The spectrum of the incident source is given in Fig. 4 over the visible wavelength range ( $380 \mathrm{~nm}$ through $780 \mathrm{~nm}$ ); the continuous curve is shown together with its approximation as a step function presenting 5 different levels. In order to define a (discrete) list of wavelengths to be traced that would be representative of the incident source spectrum, each wavelength interval determined by the step function is associated to a number of wavelengths to be considered within the particular interval, proportional to the latter's width and to the source spectrum amplitude, and given on Fig. 4 as well.

The set of wavelengths to be considered remains quite large and involves substantial time consumption for the simulation. A reduction by a factor 4 was shown not to affect the results significantly (differences lower than $2 \%$ ). 


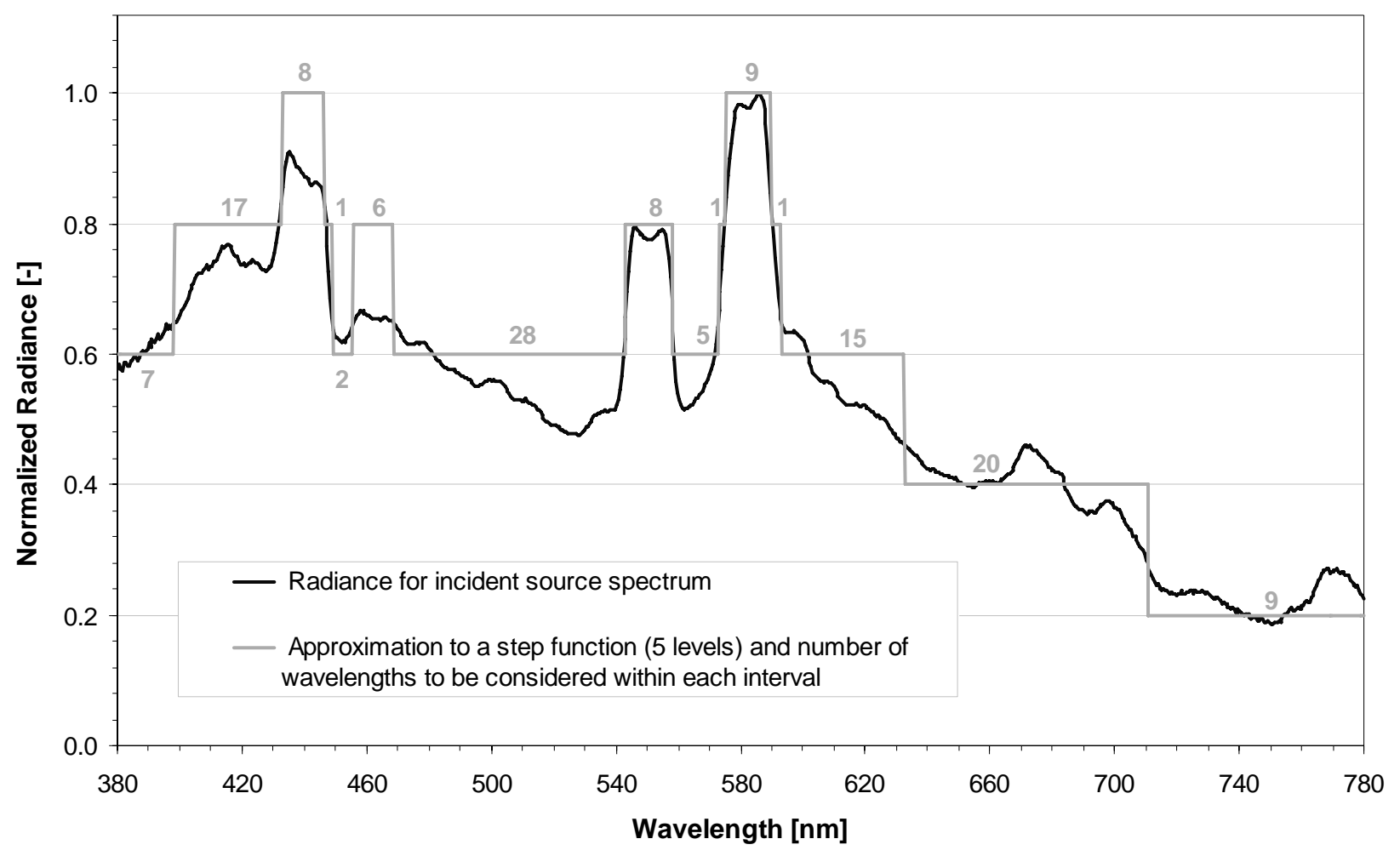

Fig. 4. Relative spectrum for the real incident source (HMI $2.5 \mathrm{~kW}$ discharge lamp mounted with Fresnel lens), and approximation by step function for a discrete wavelength set.

Instead of either moving the sample and detector according to the incidence angles, or the source itself, a virtual source was placed against the outside sample interface fitting the experimental sample diaphragm aperture (illuminated area), with rays emitted at varying angles (direction vectors) depending on the incident direction considered.

The prismatic elements were modelled according to their real geometric features (even though ideal because assumed of perfect shape). Thanks to a combination of primitive solids creation and subtractive or additive tools proposed by TRACEPRO ${ }^{\circledR}$, the respective gratings of $45^{\circ}$ slope and, with more complexity, $42^{\circ}$ and $5^{\circ}$ slopes were built (see Fig. 2). The elements were modelled as an acrylic material, according to the software's database of refractive indices (provided by manufacturers).

An opaque (100\% absorbing) diaphragm of aperture diameter respectively $6 \mathrm{~cm}$ and $10 \mathrm{~cm}$ for the symmetric and asymmetric panels was placed in front of the incident sample interface. An additional surface, presenting no interaction whatsoever with its environment, was created between the diaphragm and the sample in order to normalize light flux calculations with a reference value.

The current software features do not allow a spatial investigation of an object according to angular parameters. Instead, a set of individual detectors, associated to the different zones, was created. Practically, in order to have only one tracing session (and not 6), all the six screen positions were simulated at once by the way of 6 virtual screens in the simulation model. Each screen is split into zones using planes of azimuths $0^{\circ}, 5^{\circ}$, etc. and cones of half angles $2.5^{\circ}, 7.5^{\circ}$, 
etc. which determine the intersection lines of the zones. With an output resolution $\left(\Delta \theta_{2}, \Delta \phi_{2}\right)$ equal to $\left(5^{\circ}, 5^{\circ}\right)$, about 1400 different reception surfaces were created.

As explained in $\S 4.2$, the observed quantity is the total photometric flux received by each detection zone, easily convertible into the corresponding BTDF value through equation (2). There is therefore no need to model the reflection on a diffusing screen and the detection by the CCD camera, the calculation results being already comparable to experimental data. Furthermore, detecting the transmitted light directly on the screen allows an accurate estimation of the measurement error induced by the camera's calibration procedures (spectral, photometric, geometric, additional corrections). To avoid inter-reflections between the different detection surfaces, they were defined as perfect absorbers in the simulation model; they are shown on Fig. 5 together with the prismatic panel and the opaque diaphragm.

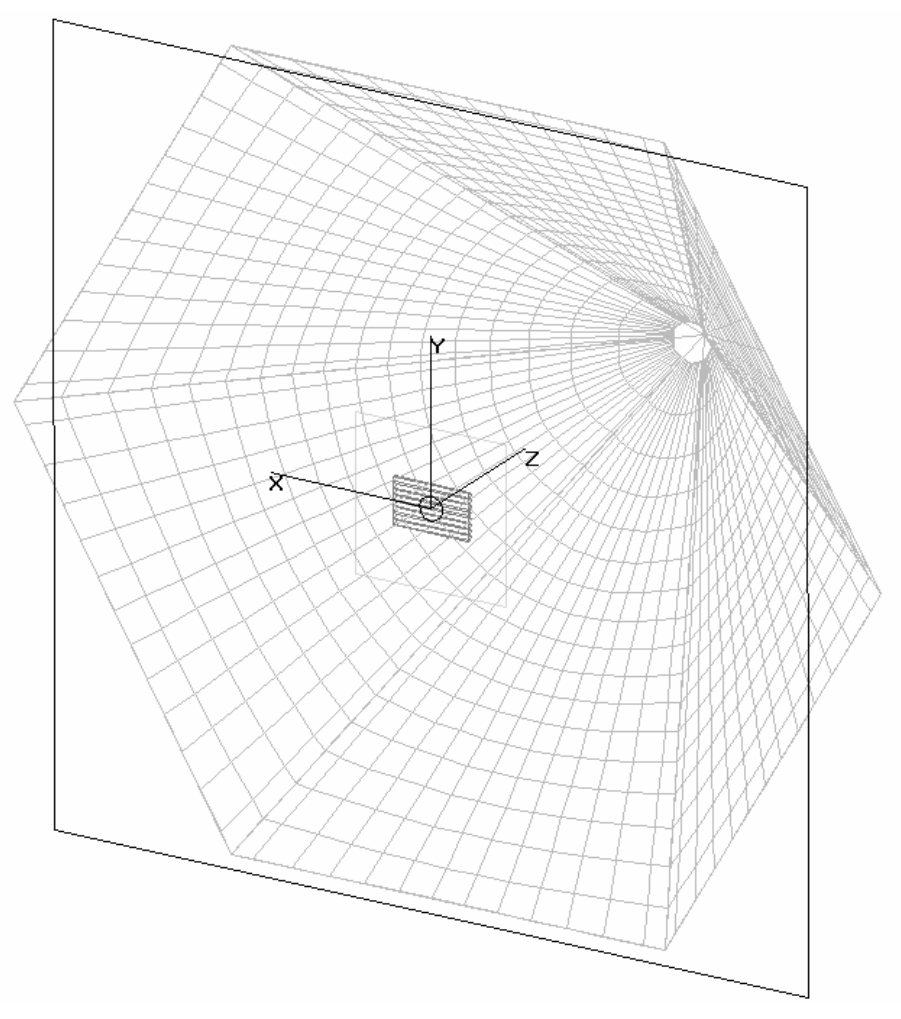

Fig. 5. Simulation model composed of an opaque diaphragm, the considered prismatic panel (sample), a noninteracting incident flux detection surface and six absorbing detection screens split into angular zones of spread $\left(\Delta \theta_{2}, \Delta \phi_{2}\right)=\left(5^{\circ}, 5^{\circ}\right)$.

\subsection{Ray-tracing results and conversion into BTDF values}

The rays were emitted from an annular grid, composed of 45 rings and sending about 200,000 rays $(\sim 6,000$ rays at each wavelength). The flux threshold (fractional value of starting flux for which a ray will be terminated) was set to 0.05 . It was checked that a larger number of rays (e.g. 15,000 per wavelength) or a lower cut-off value (e.g. 0.001) did not significantly affect the results: both induced differences lower than $1 \%$ whereas computer simulation time was considerably increased. 
A lambertian spread of $0.4^{\circ}$ (half angle) was applied to the beam for the symmetric and asymmetric panels, according to the incident source collimation characteristics for the experimental set-up (see $\S 3$ ). It must be noted that the source does not appear as a separate object in the model: it sends rays according to particular grid and beam specifications, but has no physical (optical) properties.

As explained above, the analysed quantitative output is the normalized flux $\Phi_{\text {2norm }}[\%]$ coming out from the sample and reaching a discretization zone on the screen (i.e. emitted into the solid angle $\Omega_{2}$ [sr] determined by the outgoing direction $\left(\theta_{2}, \phi_{2}\right)$ and the resolution $\left.\left(\Delta \theta_{2}, \Delta \phi_{2}\right)\right)$. The BTDF being defined as the quotient of the luminance of a surface element in a given direction by the illuminance incident on the sample, these fluxes can be converted into the associated BTDF values through equation (2), the differential quantities being approximated by their equivalent average values (Murray-Coleman and Smith, 1990):

$$
\operatorname{BTDF}\left(\theta_{1}, \phi_{1}, \theta_{2}, \phi_{2}\right)\left[s r^{-1}\right]=\frac{L_{2}}{E_{1}}=\frac{\Phi_{2}}{\Omega_{2} A \cos \theta_{2}} \cdot \frac{A}{\Phi_{1}}=\frac{\Phi_{2 \text { norm }}[\%]}{\Delta \theta_{2} \cdot \Delta \phi_{2} \cdot \sin \theta_{2} \cdot \cos \theta_{2}}
$$

where $\mathrm{L}_{2}\left[\mathrm{~cd} \mathrm{~m}^{-2}\right]$ is the luminance of the emerging (transmitted) light flux $\Phi_{2} ; \Delta \theta_{2}$ and $\Delta \phi_{2}$ are here expressed in radians.

Once converted into the corresponding BTDF values, the angles $\left(\theta_{2}, \phi_{2}\right)$ being the ones to which the zone is assigned, the data can be compared to the experimental BTDF values. Both experimental and simulated BTDF values are here assessed inside a certain angular area around the associated couples $\left(\theta_{2}, \phi_{2}\right)$, and thus depend on the output resolution $\left(\Delta \theta_{2}, \Delta \phi_{2}\right)$. They represent average values of BTDFs inside these areas, and provide a continuous - thus complete investigation of the transmitted light distribution, unlike point-per-point data that represent particular BTDF values along specific directions $\left(\theta_{2}, \phi_{2}\right)$.

\section{Results comparison}

The simulated light flux was detected in each discretization zone, converted in photometric units (lumens) and normalized to the incident flux. As the transmission features are very sharp (and therefore cover only small solid angles), the discrepancies between real and virtual values can be revealed by two-dimensional plots for varying altitudes $\theta_{2}$ and along given azimuths $\phi_{2}$, which allows to point out differences with high accuracy.

The results are shown on Figs. 6 to 11 with an output resolution $\left(\Delta \theta_{2}, \Delta \phi_{2}\right)$ of $\left(5^{\circ}, 5^{\circ}\right)$. For each analysed situation, the relevant outgoing azimuthal planes (i.e. the angles $\phi_{2}$ for which the transmission is non-zero) were determined. Both measured and calculated BTDF data were reported along these outgoing planes as functions of altitude $\theta_{2}$ for the 10 selected incident directions. For the symmetric panel (gratings $45^{\circ}$, flat face on incident side) these incident directions were $\left(40^{\circ}, 90^{\circ}\right),\left(60^{\circ}, 90^{\circ}\right),\left(24^{\circ}, 30^{\circ}\right),\left(40^{\circ}, 45^{\circ}\right)$ and $\left(60^{\circ}, 75^{\circ}\right)$. For the asymmetric panel the incident directions were $\left(20^{\circ}, 0^{\circ}\right),\left(40^{\circ}, 90^{\circ}\right),\left(0^{\circ}, 0^{\circ}\right),\left(10^{\circ}, 90^{\circ}\right)$ and $\left(20^{\circ}, 270^{\circ}\right)$, the first two being investigated with the flat face on the incident side, the others with the $42^{\circ}, 5^{\circ}$ gratings on the incident side. The azimuthal planes next to the most relevant ones were also checked (planes $\phi_{2} \pm \Delta \phi_{2}$ and $\phi_{2} \pm 2 \Delta \phi_{2}$, where $\phi_{2}$ is the azimuth angle for which the BTDFs reached their highest values) and generally revealed the same kinds of behaviours as the main plane, as shown on Figs. 6 and 10. 
A good agreement between the real and virtual BTDF values is achieved. Even though the transmission features are extremely sharp (high gradients increase the risk of having significant differences between two assessment methods), low discrepancies and a similar qualitative light behaviour can be observed, the peaks corresponding exactly to the same directions, for the main as well as the secondary maxima.

The error bars given on all the figures for the experimental and computational BTDF data are equal to $13 \%$ and $10 \%$, respectively, in relative terms.

Uncertainties due to the CCD camera calibration procedures and other corrections have been investigated thoroughly in Andersen et al. (2000); their impact on the BTDFs is 5\%. The discrepancies connected to the spatial adjustment of the facility components have been added, estimated by modelling slight variations $\left( \pm 0.5^{\circ}, \pm 2 \mathrm{~mm}\right)$ in the incident direction or detection screen position and observing the effect on the final results, which is of $8 \%$. This lead to a global error of $13 \%$ for the measurements.

The $10 \%$ relative error for the model includes the impact of the simplification hypotheses in the prism modelling (see $\S 4$ ), which was assessed by changing slightly some simulation parameters and examining how these changes affected the BTDF data. Several altered models were created for both panels and each modification was analysed individually:

- acrylic refraction indices (close to 1.49 over the most of the visible spectrum) slightly changed (average difference of 0.01)

- half a period sample position shift

- edges rounded at $0.25 \mathrm{~mm}$ off the theoretical summits

- $2 \%$ diffuse component added on prism surface to create an equivalent of surface wearing.

The relative differences on BTDF results generated by these modifications were averaged, for each studied parameter, over the set of incident and transmitted directions (only BTDF values greater than $5 \%$ of the curve maximum were considered), and their standard deviations were subtracted in order not to overestimate the possible variations of BTDF data. This lead to incertitude values of about $6 \%, 2 \%, 6 \%$ and $4 \%$, respectively associated to the refraction index, the sample position, the gratings sharpness and the diffuse component, this last parameter only affecting the results in one way (lower peaks). A global error of $10 \%$ is then obtained from calculating the square root of the sum of the squared individual relative incertitudes, including the ones due to the chosen values of threshold, number of emitted rays and the source spectrum discretization, mentioned in $\S 4$.

Figs. 6 to 11 make up a positive reciprocal validation, on one hand of the detection technique and the calibration and correction procedures, and on the other hand of the reliability and applicability of ray-tracing calculations for complex glazing assessment. 

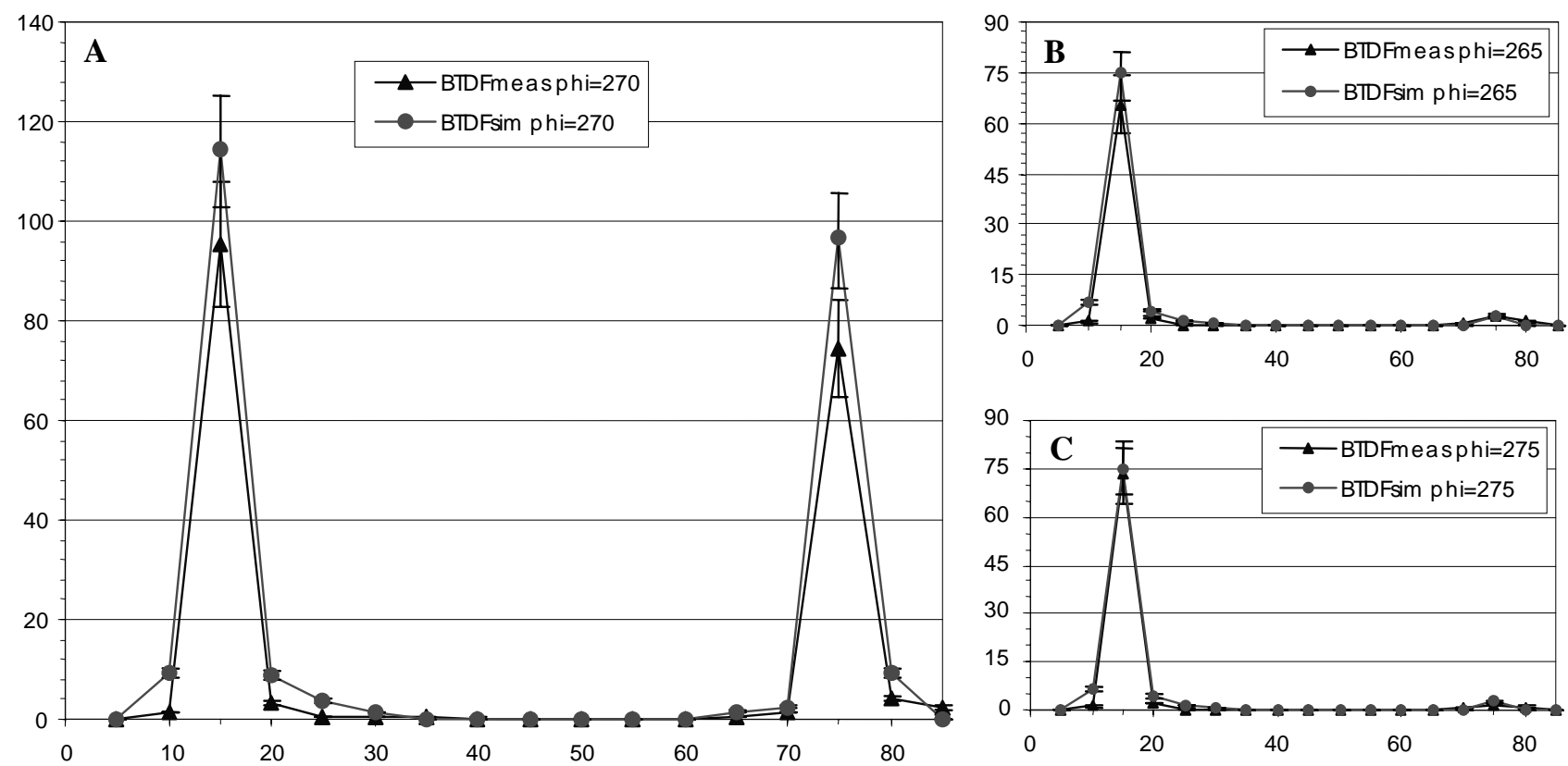

Fig. 6. BTDF $\left[\mathrm{sr}^{-1}\right]$ vs. $\theta_{2}\left[{ }^{\circ}\right]$ along $\phi_{2}$ planes: comparison of measurements (BTDFmeas) and calculations (BTDFsim) for the symmetric panel (slope $45^{\circ}$, flat face on incident side) under incidence $\left(40^{\circ}, 90^{\circ}\right.$ ). (A) Main section view, along plane $\phi_{2}=270^{\circ}$. (B) and (C) Azimuth planes next to the main one, for $\phi_{2}=265^{\circ}$ and $275^{\circ}$.
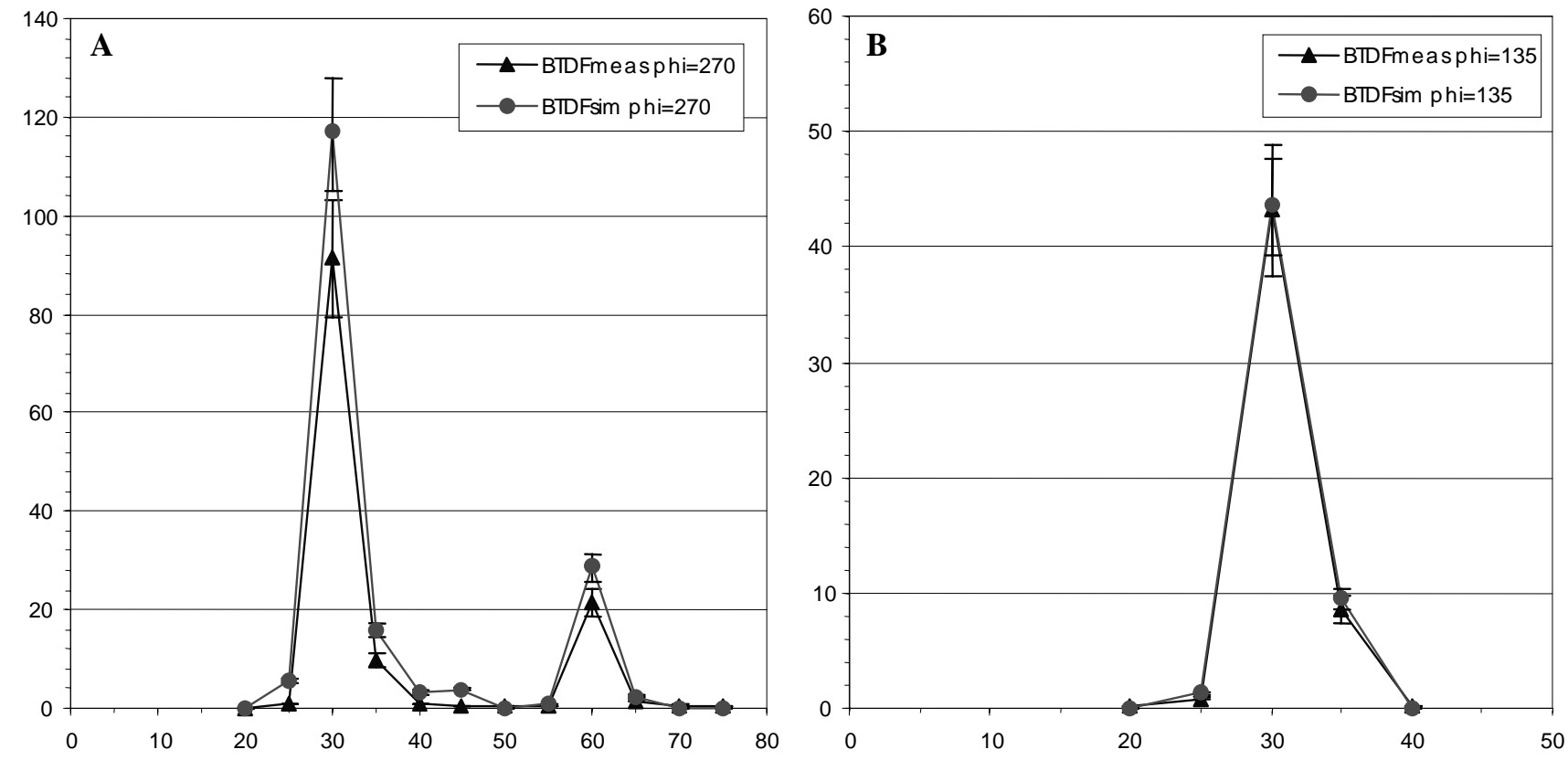

Fig. 7. BTDF $\left[\mathrm{sr}^{-1}\right]$ vs. $\theta_{2}\left[{ }^{\circ}\right]$ along $\phi_{2}$ planes: comparison of measurements (BTDFmeas) and calculations (BTDFsim) for the symmetric panel (slope $45^{\circ}$, flat face on incident side). (A) Incidence $\left(60^{\circ}, 90^{\circ}\right.$ ), main section view. (B) Incidence $\left(24^{\circ}, 30^{\circ}\right)$, main peak. 

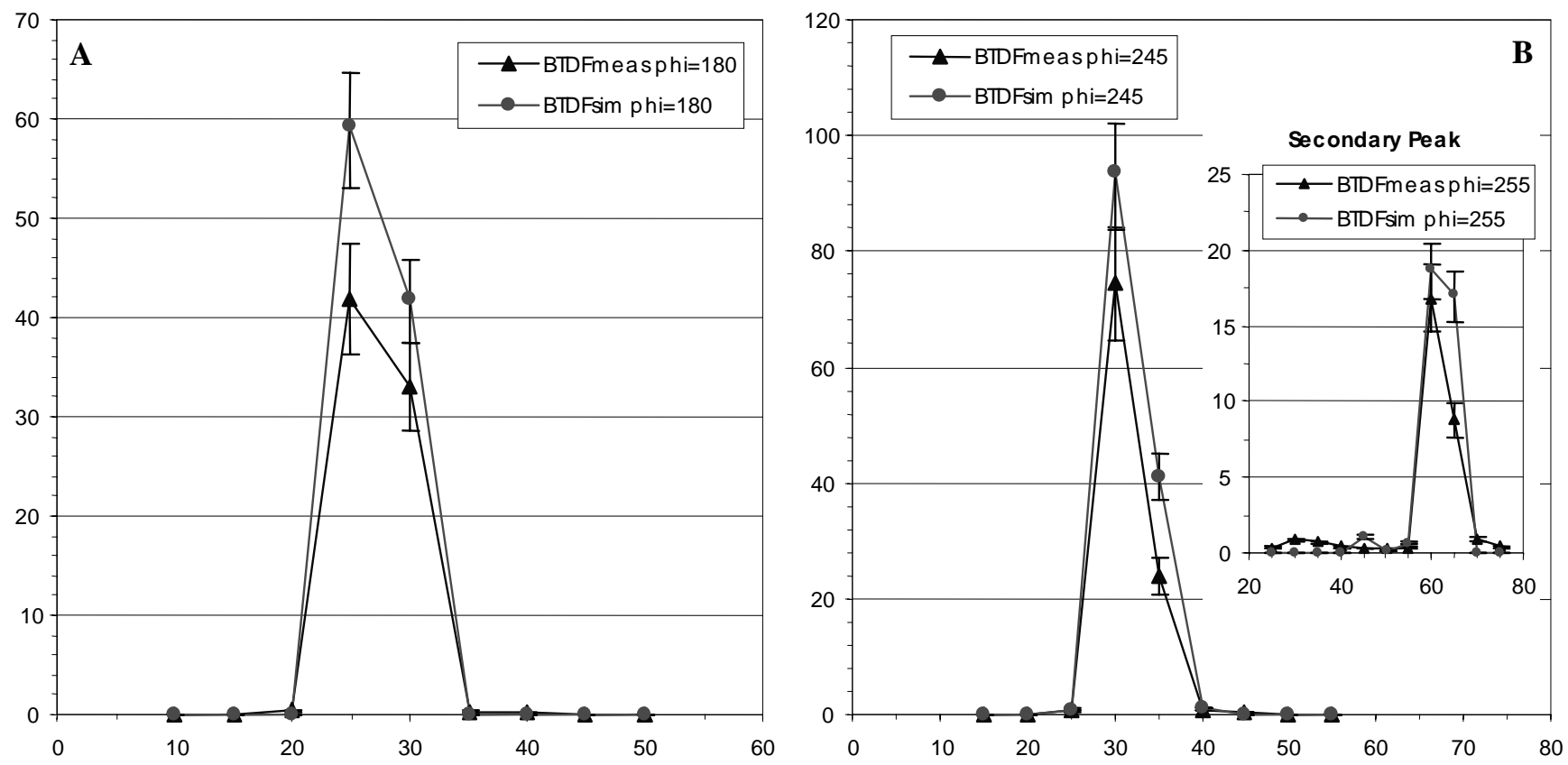

Fig. 8. BTDF $\left[\mathrm{sr}^{-1}\right]$ vs. $\theta_{2}\left[{ }^{\circ}\right]$ along $\phi_{2}$ planes: comparison of measurements (BTDFmeas) and calculations (BTDFsim) for the symmetric panel (slope $45^{\circ}$, flat face on incident side). (A) Incidence $\left(40^{\circ}, 45^{\circ}\right)$, main peak. (B) Incidence $\left(60^{\circ}, 75^{\circ}\right)$, main and secondary peaks.
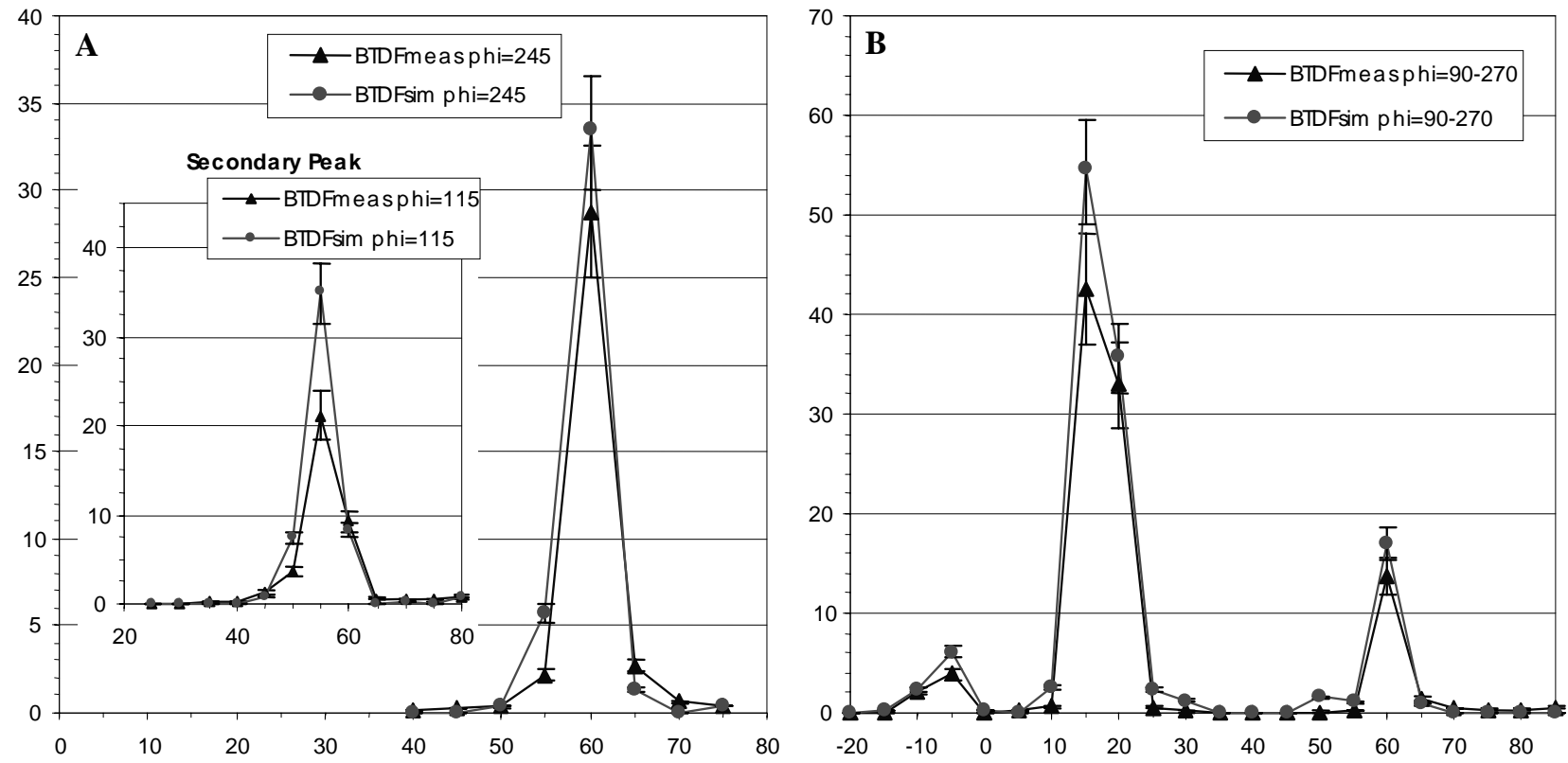

Fig. 9. BTDF $\left[\mathrm{sr}^{-1}\right]$ vs. $\theta_{2}\left[^{\circ}\right]$ along $\phi_{2}$ planes: comparison of measurements (BTDFmeas) and calculations (BTDFsim) for the asymmetric panel (slopes $42^{\circ}, 5^{\circ}$, flat face on incident side). (A) Incidence $\left(20^{\circ}, 0^{\circ}\right.$ ), main and secondary peaks. (B) Incidence $\left(40^{\circ}, 90^{\circ}\right)$, main section view showing both $\phi_{2}=90^{\circ}$ and $270^{\circ}$ for conciseness, the latter being plotted with negative values for $\theta_{2}$. 

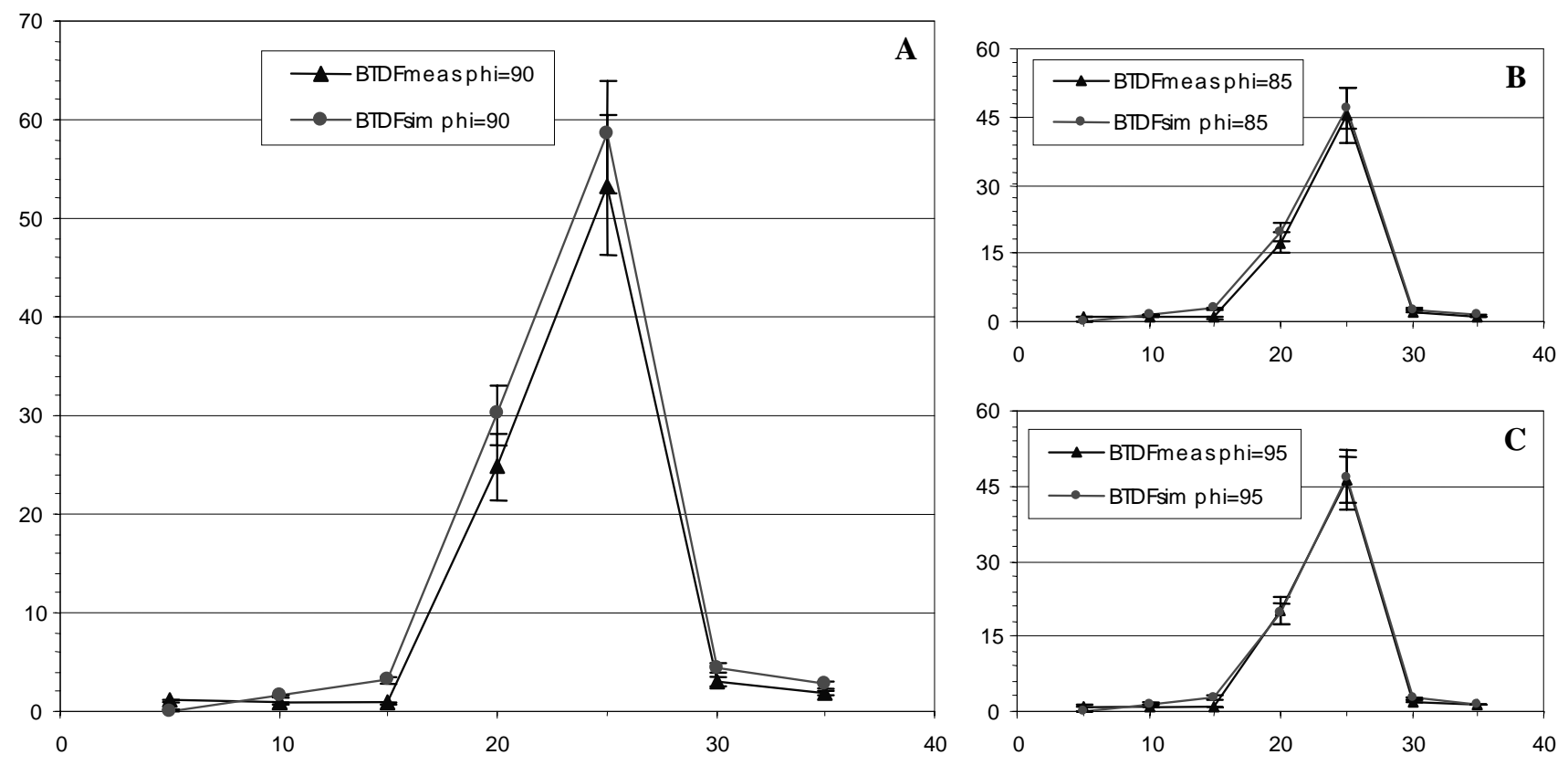

Fig. 10. BTDF $\left[\mathrm{sr}^{-1}\right]$ vs. $\theta_{2}\left[{ }^{\circ}\right]$ along $\phi_{2}$ planes: comparison of measurements (BTDFmeas) and calculations (BTDFsim) for the asymmetric panel (slopes $42^{\circ}, 5^{\circ}$, gratings on incident side) under incidence $\left(0^{\circ}, 0^{\circ}\right)$. (A) Main peak, along plane $\phi_{2}=90^{\circ}$. (B) and (C) Azimuthal planes next to the main one, for $\phi_{2}=85^{\circ}$ and $95^{\circ}$.
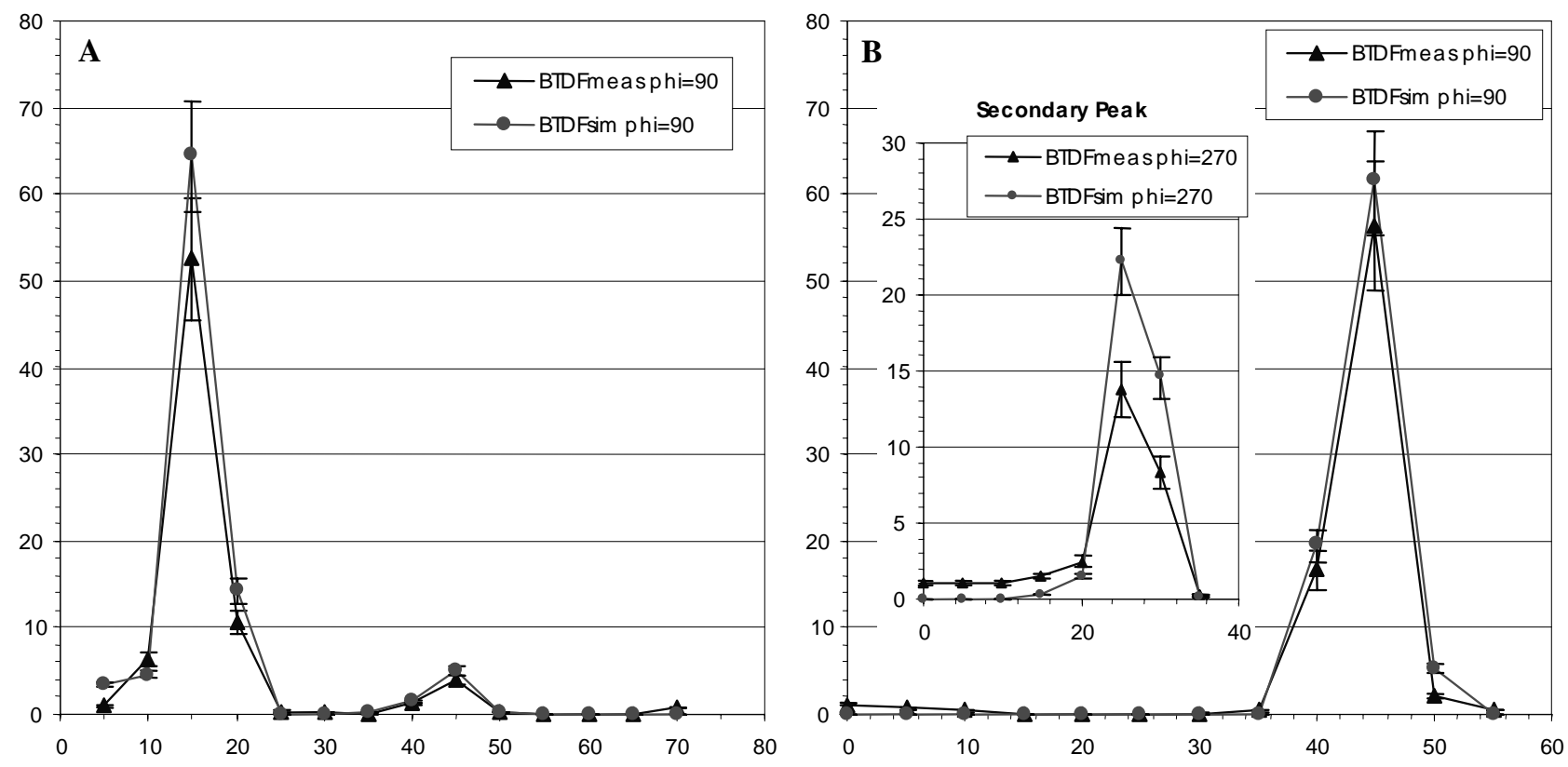

Fig. 11. BTDF $\left[\mathrm{sr}^{-1}\right]$ vs. $\theta_{2}\left[^{\circ}\right]$ along $\phi_{2}$ planes: comparison of measurements (BTDFmeas) and calculations (BTDFsim) for the asymmetric panel (slopes $42^{\circ}, 5^{\circ}$, gratings on incident side). (A) Incidence $\left(10^{\circ}, 90^{\circ}\right)$, main section view. (B) Incidence $\left(20^{\circ}, 270^{\circ}\right)$, main and secondary peaks. 


\section{Simulation of ideal experimental conditions}

Experimental BTDF data have been verified by reproducing the measurement conditions as faithfully as possible with the simulation program, in order to estimate the error due to the detection technique, i.e. to the CCD camera calibration procedures (spectral, photometric, image uniformity, etc.), the geometric relations determined between image pixels and actual outgoing directions, and the diffusing quality of the projection screen. The results presented in $\S 5$ show that these essential procedures seem to be appropriate and that the results assessed thanks to this digital imaging-based methodology are reliable.

To complement this study, an additional analysis rendered possible by the flexibility in virtual situations was carried out: the modelling of an ideal set-up, whose results could be compared to the experimental conditions.

In our case, the ideal light source would of course be the sun itself, whose particular spectrum is given on Fig. 12 in relative values over the visible range, and whose collimation is almost perfect (half-angle $0.25^{\circ}$ ).

The parameterisation of a virtual sun is realized by approximating its continuous spectrum with a discrete set of values, given on Fig. 12. A new TRACEPRO ${ }^{\circledR}$ version having been released in the meantime, the wavelengths set does not have to account for the right number of wavelengths to be simulated inside each interval to represent the spectrum (see $\S 4.1$ ); individual wavelength values to which weights are assigned are used instead, proportional to the associated radiance value. The rays are emitted according to a Lambertian distribution presenting an angular spread of $0.25^{\circ}$.

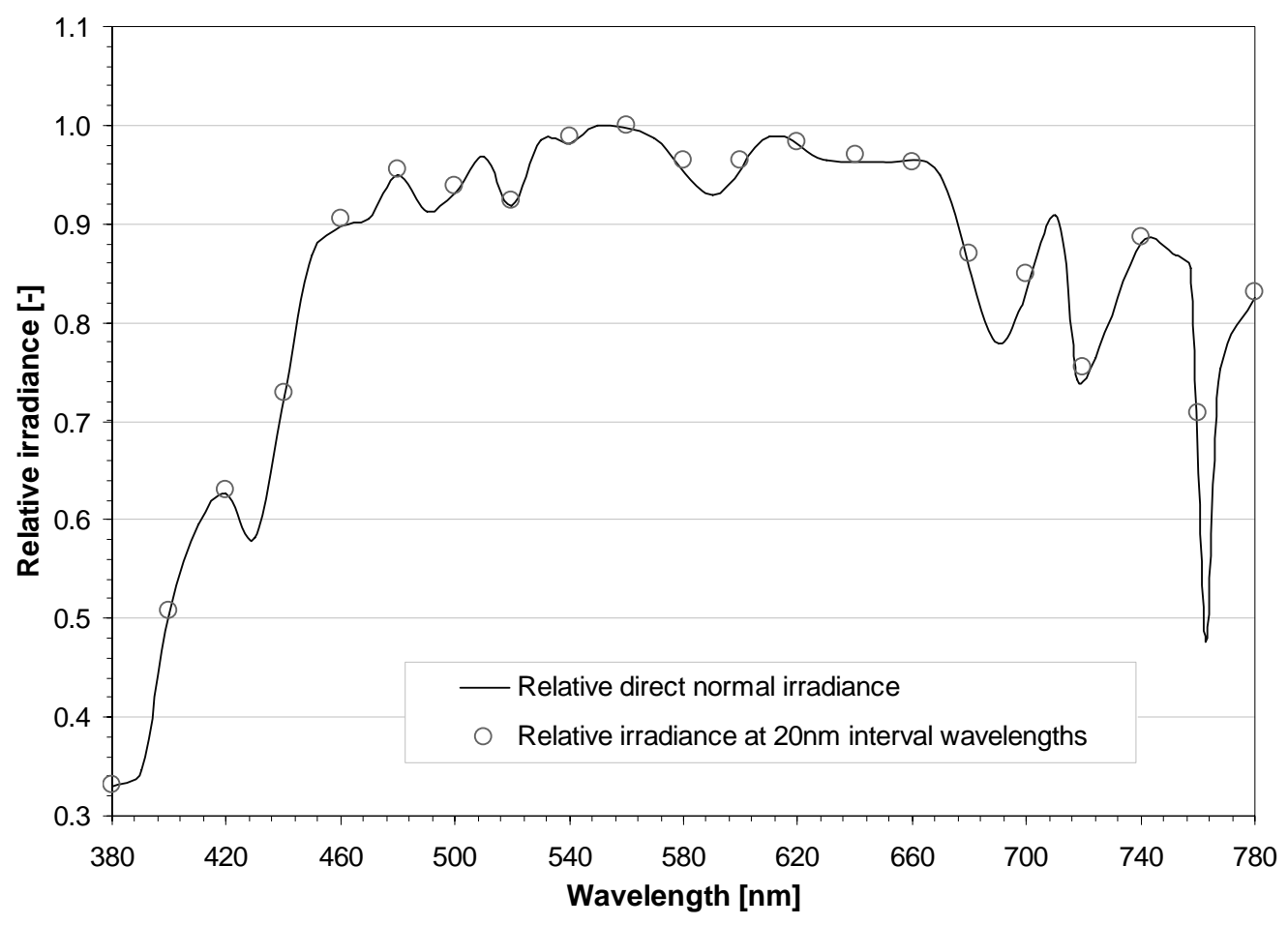

Fig. 12. Relative solar spectrum and approximation by a set of discrete values at regular wavelengths intervals, providing the weights to be assigned to each considered wavelength. 
As far as the detection surface is concerned, even though a flat projection screen is preferable to avoid any risk of inter-reflection, a virtual hemispherical surface discretized in the same way makes up a more ideal detection surface, the light being collected at a constant distance from the sample and with normal rays. Moreover, as explained in appendix A, if the source is sufficiently far away from the sample compared to the sample-to-screen distance $d\left(\theta_{2}, \phi_{2}\right)$ (which is of course the case for the sun), the transmitted light reception surface $\mathrm{A}_{\text {screen }}$ (or more generally $\mathrm{A}_{\text {screen }} \cos \alpha$ for surfaces that would not be normal to the rays) has to be comparable to the apparent illuminated area of the sample, i.e. to $A \cos \theta_{1}$.

As $\mathrm{A}$ is fixed by the diaphragm used during experimental characterization, and as the output resolution $\left(\Delta \theta_{2}, \Delta \phi_{2}\right)$ must be equal to $\left(5^{\circ}, 5^{\circ}\right)$, the only parameter that can be adjusted to fit this condition is the hemispherical detector's radius $d_{\text {hemis }}$ (distance to hemisphere). Of course, the $\left(5^{\circ}, 5^{\circ}\right)$ discretization zones surfaces vary over the hemisphere according to $\mathrm{d}_{\text {hemis }}{ }^{2} \sin \theta_{2} \Delta \theta_{2} \Delta \phi_{2}$, where $\Delta \theta_{2}$ and $\Delta \phi_{2}$ are both equal to $0.0873 \mathrm{rad}$; the calculation of $\mathrm{d}_{\text {hemis }}$ for the 6 and the $10 \mathrm{~cm}$ diaphragm diameters is therefore done by taking the average discretization zone surface over the whole hemisphere, and the obtained radii are respectively 53.5 and $89.1 \mathrm{~cm}$. These values hence provide the distance at which the detection surfaces should be positioned for an ideal BTDF characterization with specular transmission of the symmetric and asymmetric panels, according to an output resolution of $5^{\circ}$ in both altitude and azimuth. It must be observed that for the default sample diaphragm diameter $(10 \mathrm{~cm})$, the hemisphere radius is extremely close to the actual average distance from the sample to the projection screen in the experimental facility, equal to $90.5 \mathrm{~cm}$. As mentioned in $\S 2$, in order that a surface detection becomes equivalent to a directional analysis of rays emerging from a non-punctual surface, the distance between the sample and the detector should be at least ten times larger than the sample diameter, which is about the case for the determined "ideal" hemisphere radii (as well as for the experimental setup). The output referential being linked to the emerging face of the sample, the detection hemisphere is modelled with a base plane merged with the latter.

The incident directions analysed for this study are $\left(40^{\circ}, 45^{\circ}\right)$ for the symmetric panel (flat face on incident side), $\left(0^{\circ}, 0^{\circ}\right),\left(10^{\circ}, 90^{\circ}\right)$ and $\left(40^{\circ}, 90^{\circ}\right)$ for the asymmetric prism (default sample diaphragm), with gratings on incident side for the first two directions, flat face for the third. As the peaks are distributed on a small number of angular zones, only some of the zones have been created on the hemispherical detector, in order to facilitate their assignment to the corresponding angular couples $\left(\theta_{2}, \phi_{2}\right)$.

The simulation model is shown on Fig. 13 with the traced rays for the asymmetric panel, incidence $\left(40^{\circ}, 90^{\circ}\right)$. Towards the left appears the reflected part of the incident beam, not considered in this study. The figure clearly outlines the spread of transmitted rays induced by the variation of the refractive index with the wavelength, also observed for the other incidence directions (and for the experimental conditions model). The transmission peaks, revealed by Figs. 6 to 11 and 14 to 17, cannot always be identified on these ray-trace plots. The sensitivity of the human eye (photopic curve $\mathrm{V}(\lambda)$ ) is taken into account for photometric flux estimations, assigning varying weights to rays of different wavelengths. Also, the plots cannot provide quantitative information on the weight of each ray, which are all shown in the same way even though representative of very different flux values. 


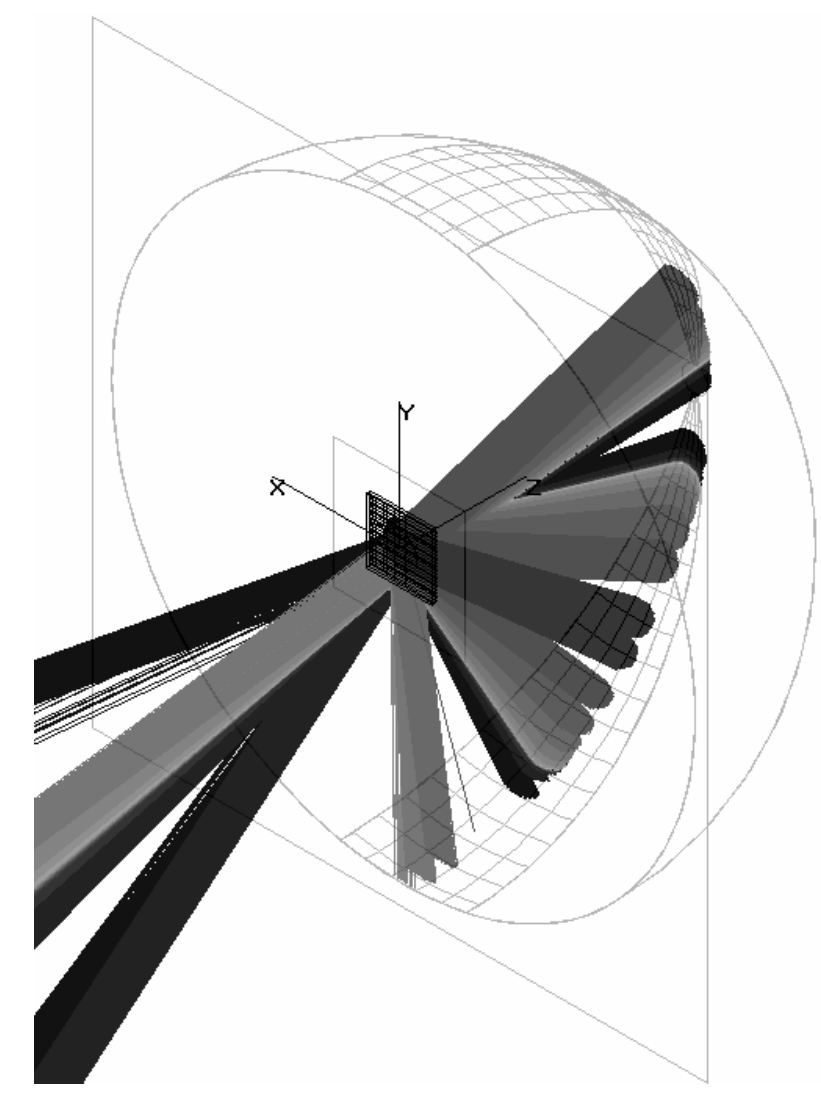

Fig. 13. Ray-tracing plots and virtual sun parameterisation for the ideal conditions simulation. Asymmetric panel (flat face on incident side), incidence $\left(40^{\circ}, 90^{\circ}\right)$.

The comparison of BTDF values obtained by measurement and by simulation with ideal conditions is given on Figs. 14 to 17. In order to appreciate the effect of changing the model only, the results provided by the simulation of experimental conditions are added on the graphs as well. The errors bars associated to the data follow the same considerations as for Figs. 6 to 11 in $\S 5$.

The observed discrepancies remain very low when comparing measurement conditions with ideal simulation results and show coherent behaviours (peaks along the same directions, similar BTDF values). The differences are generally even lower than for the experimental conditions model, which tends to prove that the new parameters (source spectrum, beam spread, detector) tend to compensate each other's effects, and that the light distribution assessment could only be improved in a slight way if using a more ideal set-up than the actual experimental facility.

One can notice that the hemisphere radius for the asymmetric panel $(89.1 \mathrm{~cm})$ is very close to the average sample to screen distance for the measurement facility $(90.5 \mathrm{~cm})$, leading to comparable average dimensions for the discretization zones. This distance being on the other hand significantly smaller for the symmetric panel hemisphere $(53.5 \mathrm{~cm})$, one can expect slightly poorer results for the latter, as observed on Fig. 14. Fortunately, the $6 \mathrm{~cm}$ diaphragm is a rather exceptional dimension, only chosen because of the physical sample's size, $10 \mathrm{~cm}$ actually being the default diaphragm for experimental assessment. 

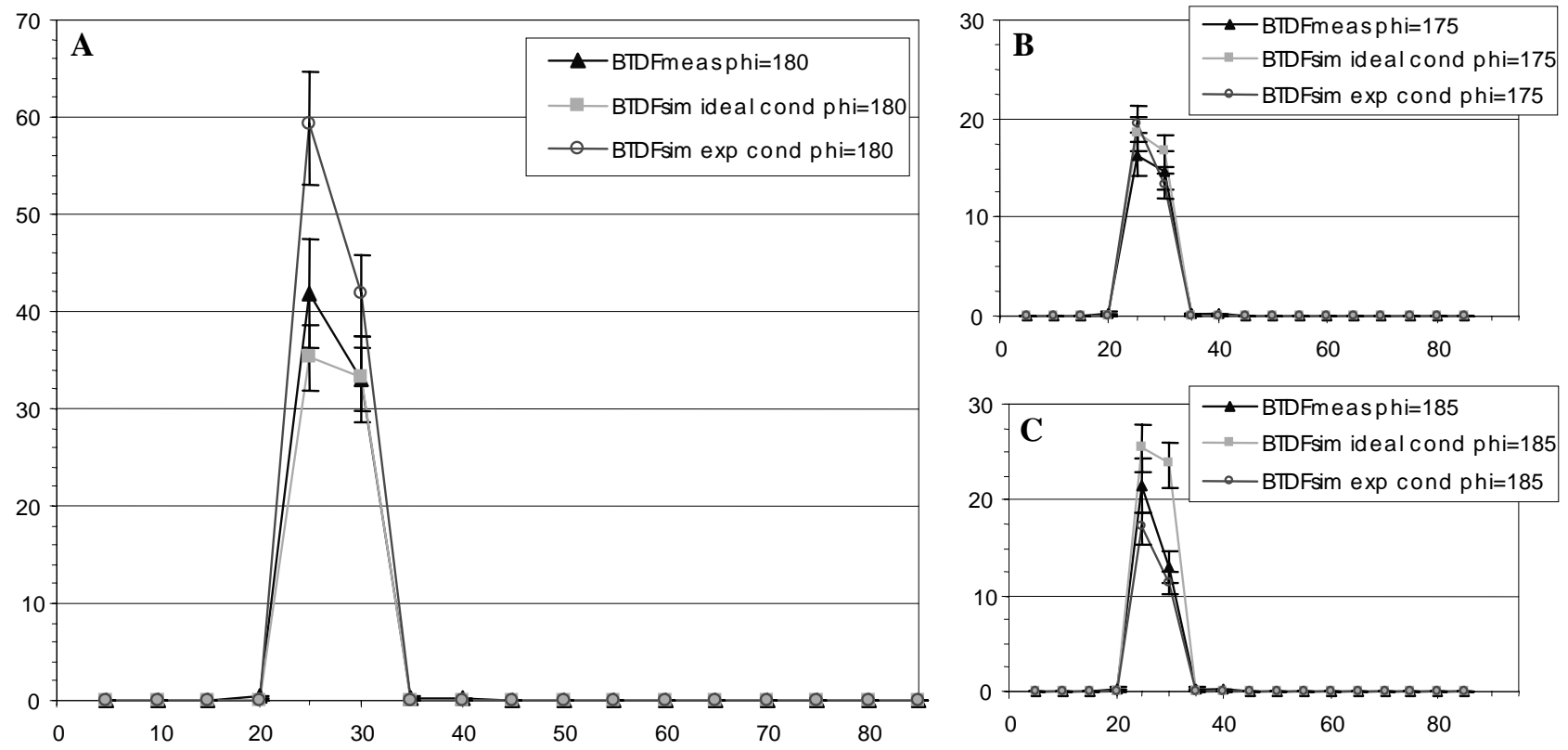

Fig. 14. BTDF [sr $\left.{ }^{-1}\right]$ vs. $\theta_{2}\left[{ }^{\circ}\right]$ along $\phi_{2}$ planes: comparison of measurements (BTDFmeas) and calculations with ideal (BTDFsim ideal) and experimental (BTDFsim exp) conditions for the symmetric panel (slope $45^{\circ}$, flat face on incident side) under incidence $\left(40^{\circ}, 45^{\circ}\right)$. (A) Main section view, along plane $\phi_{2}=180^{\circ}$. (B) and (C) Azimuth planes next to the main one, for $\phi_{2}=175^{\circ}$ and $185^{\circ}$.
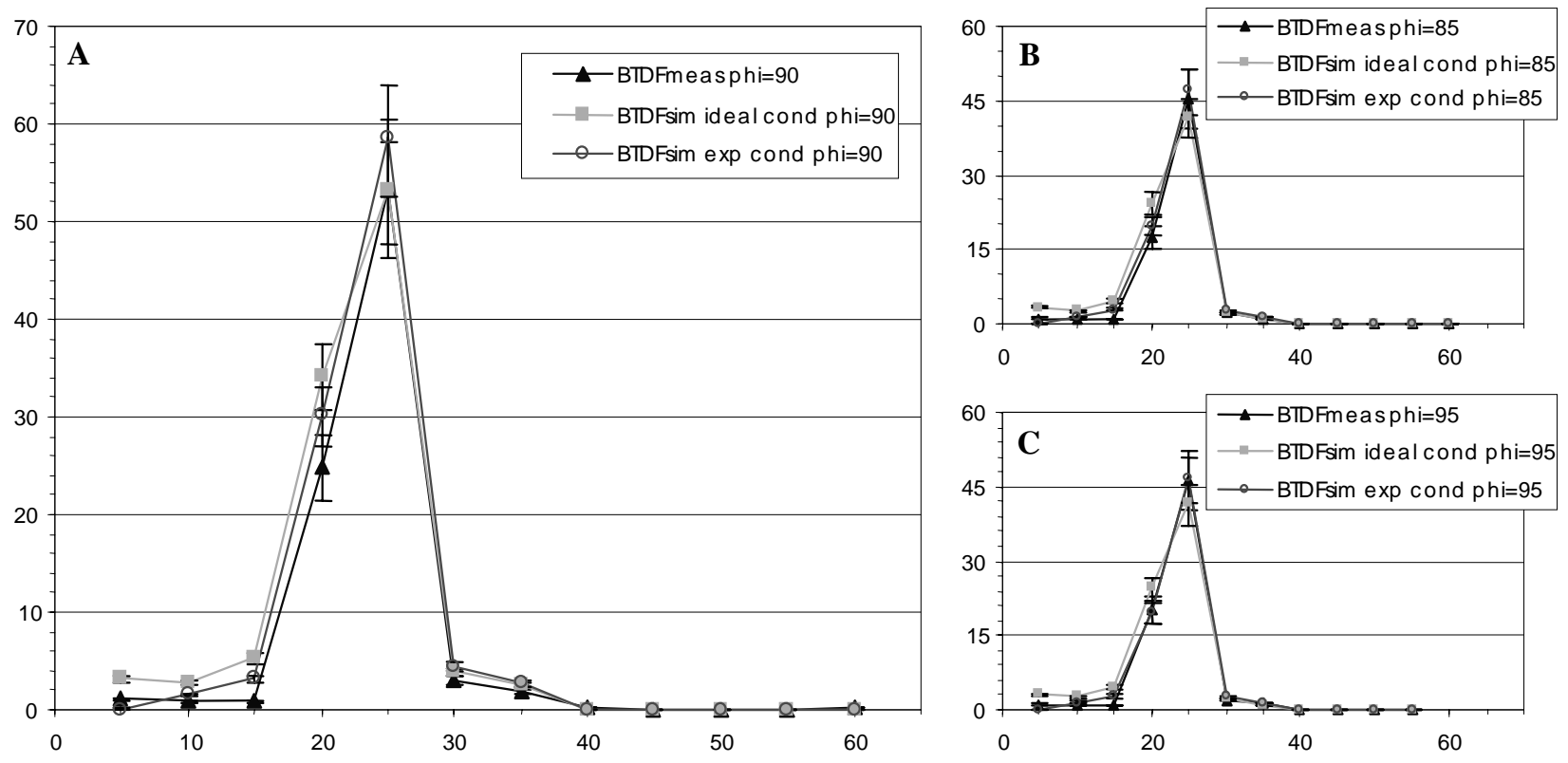

Fig. 15. BTDF [sr $\left.{ }^{-1}\right]$ vs. $\theta_{2}\left[{ }^{\circ}\right]$ along $\phi_{2}$ planes: comparison of measurements (BTDFmeas) and calculations with ideal (BTDFsim ideal) and experimental (BTDFsim exp) conditions for the asymmetric panel (slopes $42^{\circ}, 5^{\circ}$, gratings on incident side) under incidence $\left(0^{\circ}, 0^{\circ}\right)$. (A) Main section view, along plane $\phi_{2}=90^{\circ}$. (B) and (C) Azimuth planes next to the main one, for $\phi_{2}=85^{\circ}$ and $95^{\circ}$. 

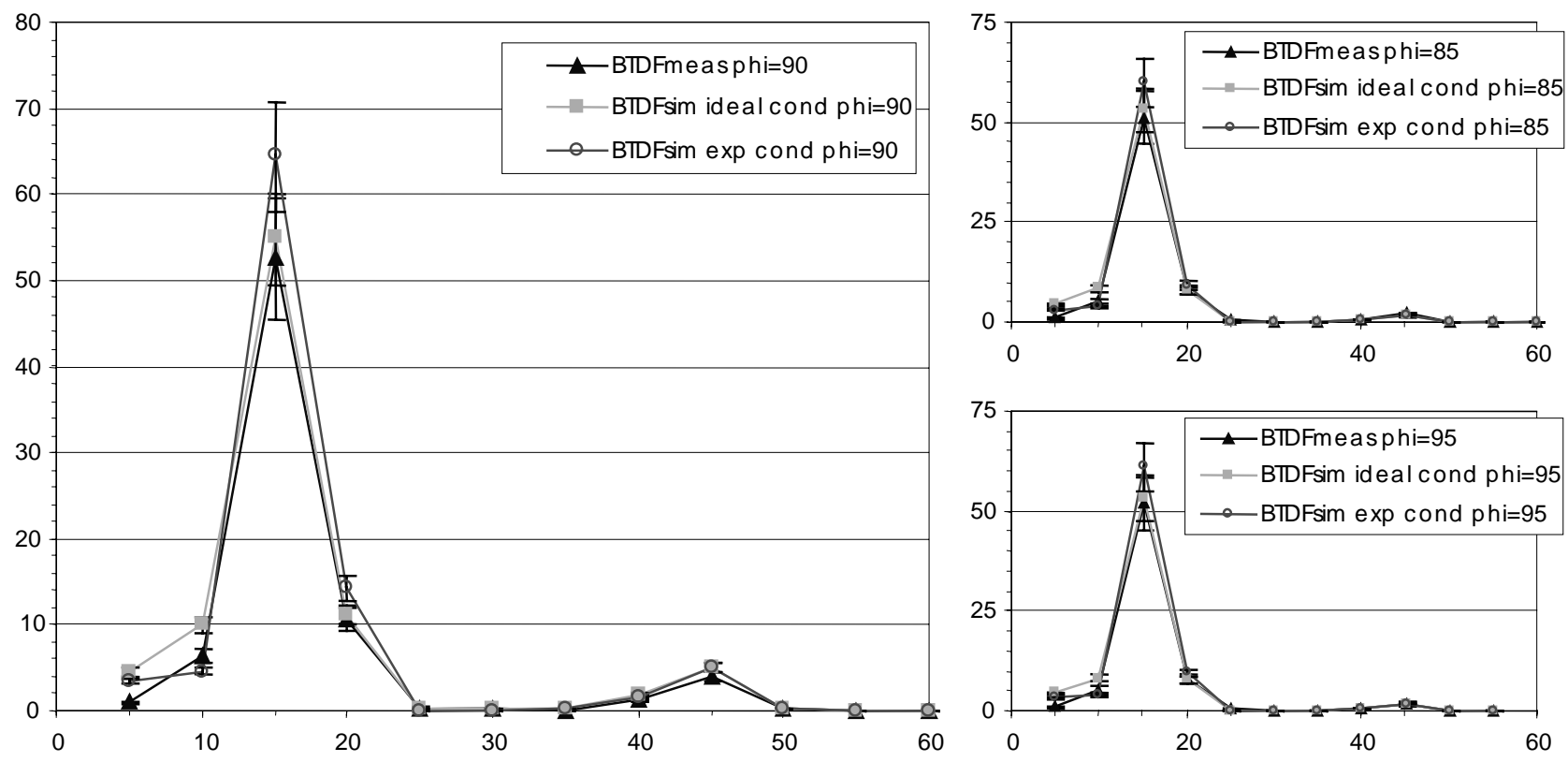

Fig. 16. BTDF [sr $\left.{ }^{-1}\right]$ vs. $\theta_{2}\left[^{\circ}\right]$ along $\phi_{2}$ planes: comparison of measurements (BTDFmeas) and calculations with ideal (BTDFsim ideal) and experimental (BTDFsim exp) conditions for the asymmetric panel (slopes $42^{\circ}, 5^{\circ}$, gratings on incident side) under incidence $\left(10^{\circ}, 90^{\circ}\right)$. (A) Main section view, along plane $\phi_{2}=90^{\circ}$. (B) and (C) Azimuth planes next to the main one, for $\phi_{2}=85^{\circ}$ and $95^{\circ}$.
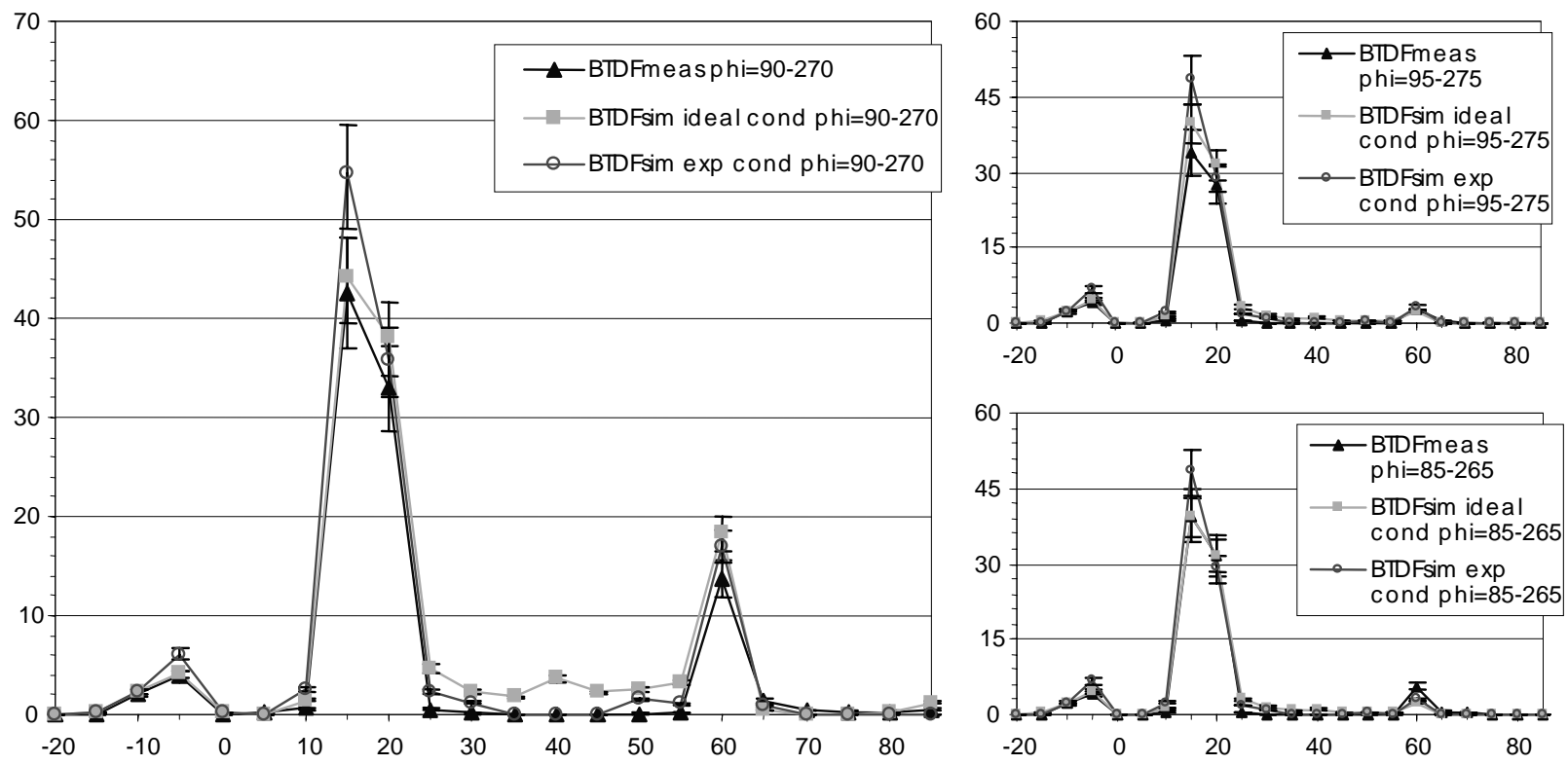

Fig. 17. BTDF [sr $\left.{ }^{-1}\right]$ vs. $\theta_{2}\left[^{\circ}\right]$ along $\phi_{2}$ planes: comparison of measurements (BTDFmeas) and calculations with ideal (BTDFsim ideal) and experimental (BTDFsim exp) conditions for the asymmetric panel (slopes $42^{\circ}, 5^{\circ}$, flat face on incident side) under incidence $\left(40^{\circ}, 90^{\circ}\right)$. (A) Main section view showing both plane $\phi_{2}=90^{\circ}$ and $270^{\circ}$ for conciseness, the latter being plotted with negative values for $\theta_{2}$. (B) and (C) Azimuth planes next to the main one, for $\phi_{2}=85^{\circ}-285^{\circ}$ and $95^{\circ}-265^{\circ}$. 


\section{Conclusion}

Monte Carlo ray-tracing simulations of prismatic light-redirecting panels produces a BTDF that is in good agreement with the BTDF measured in a photogoniometer. This agreement depends on a careful description of the physical parameters of the real equipment to create what amounts to a "virtual photogoniometer". Otherwise, agreement between measurement and calculation depends only on an accurate description of the geometry of the prismatic panel and the optical properties of the acrylic material from which the panel is made. Generally, these properties are relatively easy to specify with confidence compared to the measured properties of the complete system.

Lacking absolute standards for measurement of BTDF on full-scale systems, validation must be approached in a roundabout manner. The Monte Carlo calculation is based on first-principles and applied with algorithms that have been widely tested on a variety of optical systems. The inputs are either easily-specified geometrical descriptions or result from standardized optical measurements. Thus, for prismatic daylight-redirecting panels, the geometrical optics approach offered by Monte Carlo simulations is able to provide results with a precision sufficient for glazing systems evaluations. Conversely, the calculations agree with the BTDF measurements from a photogoniometer of carefully executed construction, which is described in some detail herein. This photogoniometer, furthermore, has been validated on simple fenestration systems of well-known properties, strengthening these comparisons.

The computational method also proved to be a valuable tool for parametric studies. First, agreement was established using the closest possible virtual copy of the physical photogoniometer. Then, more realistic parameters were set to test effects of various compromises made in the characteristics of the light source, detector screen, CCD camera and other components of the real photogoniometer. The results showed that the assumptions made in the construction of the instrument were reasonable and easily extended by calculation to even more realistic conditions.

The importance of these results goes beyond validation of the specific glazing and instrument of this study. We deliberately chose a glazing system that would be difficult to reproduce in some aspects. It is plausible, therefore, that this method will be quite general and could reduce the burden of difficult and time-consuming measurements on complex systems. Also, when further confidence in this approach has been established, validation will be facilitated among the disparate and incomparable measurement systems worldwide.

\section{Acknowledgements}

Marilyne Andersen was supported by the Swiss National Science Foundation, fellowship 81EL66225, during her stay at the Lawrence Berkeley National Laboratory. The project was supported by the Assistant Secretary for Energy Efficiency and Renewable Energy, Office of Building Technology, State and Community Programs, Office of Building Research and Standards of the U.S. Department of Energy under Contract No. DE-AC03-76SF00098. 
The authors wish to thank Dr. Joseph Klems at LBNL for his enlightening advices in bidirectional distribution functions assessment, and Dr. Ross McCluney at the Florida Solar Energy Center for sharing his background in ray-tracing simulations.

\section{Appendix A. Including of specular component in a BTDF assessment}

As mentioned in $\S 2$, and illustrated by Fig. A.1A, the analytical expressions for BTDFs differ whether they are related to the specular or the diffuse component of the transmitted light. The specular part is not related to a solid angle, and varies with the distance from source to detector, whereas the diffuse part depends on the considered solid angle, and therefore appears as a function of the distance from sample to detector (see equation (1)).

By expressing both specular and diffuse BTDFs and comparing their associated equations, one can find out what conditions would be necessary for them to be considered as equivalent, and therefore for accepting to measure both components together.

The BTDF is defined as "the quotient of the luminance of a surface element in a given direction, by the illuminance incident on the sample" (CIE, 1977). Because the illuminance is independent of which component is chosen in the transmitted light, one can analyse directly the expressions of luminance emerging from the sample for specular and diffuse light. In the case of the photogoniometer considered in this paper, it would actually be preferable to compare the expressions of luminance emitted by the projection screen and detected by the CCD camera, a quantity that is determinant in the BTDF assessment, schematised by Fig. A.1B.
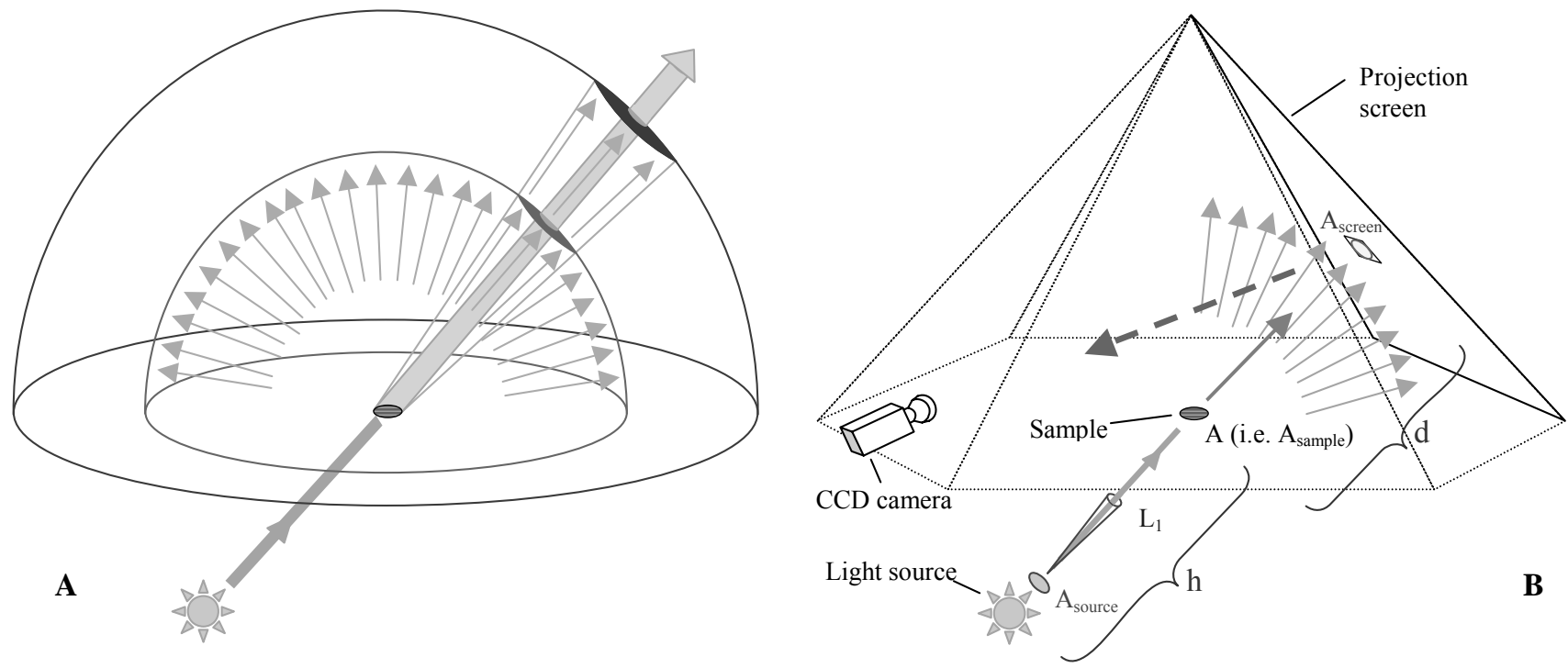

Fig. A.1. Detection of the light transmitted through a sample. (A) Specular component against diffuse transmission. (B) Light transmission and detection with the digital imaging-based photogoniometer.

Equations (A.1) and (A.2) respectively describe the luminance emitted from the screen due to direct (specular) transmission ( $\left.\mathrm{L}_{\text {screen_spec }}\right)$ and to diffuse transmission ( $\mathrm{L}_{\text {screen_diff }}$, the latter being deduced from equation (1). Both definitions require the projection screen to be of lambertian type, which has been shown in Andersen et al. (2000) to be a very reasonable assumption. The 
formal differential quantities are replaced by their equivalent average values (Murray-Coleman and Smith, 1990):

$$
\begin{aligned}
& L_{\text {screen_spec }}=\tau \cdot \frac{\rho}{\pi} \cdot \frac{h^{2} \cos \alpha}{(h+d)^{2} \cos \theta_{1}} E_{1} \\
& L_{\text {screen_diff }}=\frac{\rho}{\pi} \cdot \frac{L_{2} \cdot A \cdot \cos \theta_{2} \cdot \cos \alpha}{d^{2}}
\end{aligned}
$$

where $\mathrm{h}$ is distance from source to sample and $\tau$ is hemispherical light transmittance, in this case only related to direct transmittance. The other quantities are defined according to the same nomenclature as in equations (1) and (2).

If the specular and the diffuse parts of the transmitted light are not separated in the measurement, inducing that quantities $\mathrm{L}_{\text {screen_spec }}$ and $\mathrm{L}_{\text {screen_diff }}$ are converted likewise into BTDF data, expressions (A.1) and (A.2) must be equivalent under the actual experimental conditions.

This leads to relation (A.3) to be verified:

$$
L_{2} \approx \frac{d^{2} h^{2}}{(h+d)^{2}} \cdot \frac{1}{A \cdot \cos \theta_{2} \cdot \cos \theta_{1}} \cdot \tau \cdot E_{1}
$$

Replacing $\mathrm{E}_{1}$ by its definition as a function of luminance, i.e. applying equation (A.4):

$$
E_{1}=L_{1} \cdot \cos \theta_{1} \cdot \Omega_{1}
$$

where $L_{1}$ is the luminance of the incoming light flux and $\Omega_{1}$ its associated solid angle, expressed by (A.5), $\mathrm{A}_{\text {source }}$ being the source area (considered as planar) sending rays towards $\mathrm{A}$ :

$$
\Omega_{1}=\frac{A_{\text {source }}}{h^{2}}
$$

we obtain relation (A.6):

$$
L_{2} \approx \frac{d^{2}}{(h+d)^{2}} \cdot \frac{A_{\text {source }}}{A \cdot \cos \theta_{2}} \cdot \tau \cdot L_{1}
$$

Expressing $\mathrm{L}_{1}, \mathrm{~L}_{2}$ and $\tau$ by their formal definitions (still in average quantities), given by equations (A.7):

$$
L_{1}=\frac{\Phi_{1}}{A_{\text {source }} \cdot \Omega_{1} \cdot \cos \theta_{1}} \quad L_{2}=\frac{\Phi_{2}}{A \cdot \Omega_{2} \cdot \cos \theta_{2}} \quad \tau=\frac{\Phi_{2}}{\Phi_{1}}
$$

we can rewrite relation (A.6) into (A.8):

$$
\frac{1}{\Omega_{2}} \approx \frac{d^{2}}{(h+d)^{2}} \cdot \frac{1}{\Omega_{1} \cdot \cos \theta_{1}}
$$

As the incident beam is considered perfectly collimated in the BTDF definition, and provided that the source is larger than the sample (which is the case for the chosen experimental set-up), the emitting area $A_{\text {source }}$ that actually sends rays towards the sample area $\mathrm{A}$ is in fact equivalent to the latter. According to (A.5) and to the solid angle definition for $\Omega_{2}$, we can thus write equations (A.9):

$$
\Omega_{1}=\frac{A}{h^{2}} \quad \Omega_{2}=\frac{A_{\text {screen }} \cdot \cos \alpha}{d^{2}}
$$


This finally leads to the conditions that have to be fulfilled with the digital imaging-based photogoniometer for assessing both specular and diffuse light transmission properties together, which are expressed by relation (A.10): the ratio of squared distances from sample to source and from detector to source must be comparable to the ratio of the apparent surfaces of the sample and the averaging (discretization) zone, apparent in the sense of being seen respectively along the incident and emerging directions.

$$
\frac{h^{2}}{(h+d)^{2}} \approx \frac{A \cdot \cos \theta_{1}}{A_{\text {screen }} \cdot \cos \alpha}
$$

For the experimental facility considered in this paper, the distance $\mathrm{h}$ from sample to light source is equal to $6.5 \mathrm{~m}$; the average distance $\mathrm{d}$ from sample to screen being of $0.905 \mathrm{~m}$, we obtain a distance ratio of 0.77 .

As mentioned in $\S 2$, the output resolution must be determined by the sample size. Different criteria are to be followed, and their compromise leads to the determination of the most suitable steps $\Delta \theta_{2}$ and $\Delta \phi_{2}$.

The most important one is to have discretization zones of apparent dimensions similar to the apparent diaphragm aperture, in order to get reliable BTDF values, which follows condition (A.10) for a sufficiently distant source position.

The other ones are, on one hand, to choose zones angular expanses close to the possible divergence in ray directions emerging from the non-punctual sample and reaching a given point in order to compensate this effect by averaging the values, and on the other hand, to ensure that an entire discretization zone is comprised inside each luminous peak in order to guarantee the extraction of the maximal value of BTDFs after averaging them inside the zones, this last criteria being of much less importance than the others.

Taking the default set of 145 incident directions (following the sky discretization proposed by Tregenza (1987) as mentioned in $\S 3$ ) and the default sample diaphragm diameter (equal to 10 $\mathrm{cm})$, an average value for $A \cos \theta_{1}$ can be determined. The output resolution $\left(\Delta \theta_{2}, \Delta \phi_{2}\right)$ advised for this sample area being of $\left(5^{\circ}, 5^{\circ}\right)$ and the screen position being fixed and known, thus allowing to calculate the dimensions of $\mathrm{A}_{\text {screen }}$ for every output discretization zone, one can calculate the average value for $\mathrm{A}_{\text {screen }} \cos \alpha$.

The ratio of the two average apparent areas is 1.01, which proves that the first criteria for choosing the output resolution is closely followed, and provides an almost perfect respect of condition (A.10) if the source distance is sufficient for the ratio $\mathrm{h}^{2} /(\mathrm{h}+\mathrm{d})^{2}$ to approach one.

This is only nearly the case for the source position in the considered experimental set-up (the source has recently been replaced by a more efficient one, which is now positioned at a greater distance from the sample), and there is still a difference of $24 \%$ between distance and area ratios of condition (A.10). However, as observed in $\S 6$, the impact on the BTDF values is by far less significant, as outlined by the comparisons of the ideal model (where the condition is respected, the hemisphere radius having been calculated in order that the discretization zones average area is equal to the average value of $A \cos \theta_{1}$ ) with the experimental situation for the default diaphragm.

This tends to show that the verification of condition (A.10) for the actual photogoniometer set-up can be considered sufficient to accept specular transmission to be measured together with diffuse light. 


\section{References}

Andersen M., Scartezzini J.-L., Roecker C., Michel L. (2000). Bi-directional Photogoniometer for the Assessment of the Luminous Properties of Fenestration Systems. CTI Project No. 3661.2, LESO-PB/EPFL (Ed), Lausanne.

Andersen M., Michel L., Roecker C., Scartezzini J.-L. (2001). Experimental assessment of bidirectional transmission distribution functions using digital imaging techniques. Energy and Buildings 33 (5), 417-431.

Andersen M. (2001). Use of matrices for the adaptation of video-based photogoniometric measurements to a variable referential. In Proceedings of Solar Energy in Buildings CISBAT'01, EPFL (Ed), pp. 193-198, Lausanne, Switzerland.

Andersen M. (2002). Light distribution though advanced fenestration systems. Building Research and Information 30 (4), 264-281.

Apian-Bennewitz P. (1994). Designing an apparatus for measuring bidirectional reflection/transmission. SPIE 0-8194-1564-2, 2255, 697-706.

Aydinli S. (1999). Measurement of Luminous Characteristics of Daylighting Materials. IEA SHCP Task 21 / ECBCS Annex 29, TUB (Ed), Berlin.

Bakker L., van Dijk D. (1995). Measuring and processing optical transmission distribution functions of TI-materials. Private communication, TNO Building and Construction Research (Ed), Delft.

Breitenbach J., Rosenfeld J.L.J. (1998). Design of a Photogoniometer to Measure Angular Dependent Optical Properties. In Proceedings of International Conference on Renewable Energy Technologies in Cold Climates, Solar Energy Society of Canada Inc. (Ed), pp. 386-391, Ottawa, Canada.

Campbell N.S (1998). A Monte Carlo approach to thermal radiation distribution in the built environment. PhD thesis, University of Nottingham (Ed), Nottingham.

CIE Commission Internationale de l'Eclairage (1977). Radiometric and photometric characteristics of materials and their measurement. CIE 38 (TC-2.3).

Compagnon R. (1994). Simulations numériques de systèmes d'éclairage naturel à pénétration latérale. PhD Thesis, EPFL (Ed), Lausanne.

van Dijk D. (2001). Daylighting Products with Redirecting Visual Properties. JOE3-CT98-0096 REVIS Draft Publishable Final Report, TNO (Ed), Delft.

Kuhn T.E., Buehler C., Platzer W.J. (2000). Evaluation of overheating protection with sunshading systems. Solar Energy 69 (Suppl. 1-6), 59-74. 
Mitanchey R., Periole G., Fontoynont M. (1995). Goniophotometric measurements: Numerical simulation for research and development applications. Lighting Research and Technology 27 (4), 189-196.

Molina J.L., Coronel J.F., Maestre I.R., Guerra J.J. (1995). Optical and thermal modeling of complex glazing systems. 6th experts meeting of IEA SHC Task 18 / Advanced glazing and associated materials for solar and buildings applications, University of Seville (Ed), Seville.

Murray-Coleman J.F., Smith A.M. (1990). The Automated Measurement of BRDFs and their Application to Luminaire Modeling. Journal of the Illuminating Engineering Society 19 (1), 8799.

Papamichael K., Klems J., Selkowitz S. (1988). Determination and Application of Bidirectional Solar-Optical Properties of Fenestration Materials. LBL-25124, Lawrence Berkeley Laboratory (Ed), Berkeley.

Tregenza P.R. (1987). Subdivision of the sky hemisphere for luminance measurements. Lighting Research and Technology 19. 